\title{
Ce(III)-based Frameworks: From 1D Chain to 3D Porous Metal-Organic Framework
}

Parviz Gohari Derakhshandeh*, Sara Abednatanzi, Karen Leus, Jan Janczak, Rik Van Deun, Pascal Van Der Voort, Kristof Van Hecke*

Table S1. Selected bond lengths $[\AA]$ and angles $\left[^{\circ}\right]$ for $\mathbf{1}, \mathbf{2}$ and $\mathbf{3}$

\begin{tabular}{|c|c|c|c|}
\hline 1 & & & \\
\hline $\mathrm{Ce} 1-\mathrm{O} 1$ & $2.410(4)$ & $\mathrm{Ce} 1-\mathrm{O} 10$ & $2.505(4)$ \\
\hline $\mathrm{Ce} 1-\mathrm{O} 5$ & $2.456(4)$ & Ce1-O11 & $2.555(4)$ \\
\hline $\mathrm{Ce} 1-\mathrm{O}^{i}$ & $2.547(4)$ & $\mathrm{Ce} 1-\mathrm{O} 12$ & $2.614(4)$ \\
\hline $\mathrm{Ce} 1-\mathrm{O}^{i i}$ & $2.540(4)$ & $\mathrm{Ce} 1-\mathrm{N} 2^{i}$ & $2.713(4)$ \\
\hline Ce1-O9 & $2.555(4)$ & & \\
\hline $\mathrm{O} 1-\mathrm{Ce} 1-\mathrm{O} 5$ & $82.44(12)$ & $\mathrm{O} 7^{i}-\mathrm{Ce} 1-\mathrm{O} 10$ & $111.91(12)$ \\
\hline $\mathrm{O} 1-\mathrm{Ce} 1-\mathrm{O} 9$ & 76.64(13) & $\mathrm{O} 7^{i}-\mathrm{Ce} 1-\mathrm{N} 2^{i}$ & $59.57(12)$ \\
\hline $\mathrm{O} 1-\mathrm{Ce} 1-\mathrm{O} 10$ & $138.55(13)$ & $\mathrm{O} 7^{i}-\mathrm{Ce} 1-\mathrm{O}^{i i}$ & $130.17(11)$ \\
\hline $\mathrm{O} 1-\mathrm{Ce} 1-\mathrm{O} 11$ & $77.10(13)$ & $\mathrm{O} 8{ }^{i i-} \mathrm{Ce} 1-\mathrm{O} 9$ & $69.88(12)$ \\
\hline $\mathrm{O} 1-\mathrm{Ce} 1-\mathrm{O} 12$ & $74.36(13)$ & $\mathrm{O} 8^{i i-} \mathrm{Ce} 1-\mathrm{O} 10$ & $67.17(12)$ \\
\hline $\mathrm{O} 1-\mathrm{Ce} 1-\mathrm{O} 7^{i}$ & $69.80(12)$ & $\mathrm{O} 8^{i i}-\mathrm{Ce} 1-\mathrm{N} 2^{i}$ & $74.60(13)$ \\
\hline $\mathrm{O} 1-\mathrm{Ce} 1-\mathrm{N} 2^{i}$ & $128.86(13)$ & $\mathrm{O} 8^{i i-} \mathrm{Ce} 1-\mathrm{O} 12$ & $137.02(12)$ \\
\hline $\mathrm{O} 1-\mathrm{Ce} 1-\mathrm{O} 8^{i i}$ & $145.18(12)$ & $\mathrm{O} 8^{i i-} \mathrm{Ce} 1-\mathrm{O} 11$ & $98.48(14)$ \\
\hline O5-Ce1-O9 & $72.52(12)$ & $\mathrm{O} 9-\mathrm{Ce} 1-\mathrm{O} 10$ & $113.74(13)$ \\
\hline $\mathrm{O} 5-\mathrm{Ce} 1-\mathrm{O} 10$ & $138.83(12)$ & O9-Ce1-O11 & $68.11(13)$ \\
\hline
\end{tabular}




$\begin{array}{cccc}\mathrm{O} 5-\mathrm{Ce} 1-\mathrm{O} 11 & 138.75(13) & \mathrm{O} 9-\mathrm{Ce} 1-\mathrm{O} 12 & 132.56(12) \\ \mathrm{O} 5-\mathrm{Ce} 1-\mathrm{O} 12 & 137.65(13) & \mathrm{O} 9-\mathrm{Ce} 1-\mathrm{N} 2^{i} & 136.67(12) \\ \mathrm{O} 5-\mathrm{Ce} 1-\mathrm{O} 7^{i} & 73.18(12) & \mathrm{O} 10-\mathrm{Ce} 1-\mathrm{O} 11 & 71.21(12) \\ \mathrm{O} 5-\mathrm{Ce} 1-\mathrm{N} 2^{i} & 76.99(12) & \mathrm{O} 10-\mathrm{Ce} 1-\mathrm{O} 12 & 69.92(13) \\ \mathrm{O} 5-\mathrm{Ce} 1-\mathrm{O} 8^{i i} & 78.76(12) & \mathrm{O} 10-\mathrm{Ce} 1-\mathrm{N} 2^{i i} & 72.33(12) \\ \mathrm{O} 7^{i}-\mathrm{Ce} 1-\mathrm{O} 12 & 65.76(12) & \mathrm{O} 11-\mathrm{Ce} 1-\mathrm{N} 2^{i} & 142.59(12) \\ \mathrm{O} 7^{i-\mathrm{Ce} 1-\mathrm{O} 11} & 129.53(13) & \mathrm{O} 11-\mathrm{Ce} 1-\mathrm{O} 12 & 69.33(14) \\ \mathrm{O}^{i}{ }^{i} \mathrm{Ce} 1-\mathrm{O} 9 & 134.35(12) & \mathrm{O} 12-\mathrm{Ce} 1-\mathrm{N} 2^{i} & 90.56(12)\end{array}$

Symmetry codes: (i) 1-x,1-y,1-z; (ii) 2-x,1-y,1-z;

\begin{tabular}{|c|c|c|c|}
\hline 2 & & & \\
\hline $\mathrm{Ce} 1-\mathrm{O} 1$ & $2.501(3)$ & $\mathrm{Ce} 1-\mathrm{O} 7^{i i}$ & $2.495(3)$ \\
\hline $\mathrm{Ce} 1-\mathrm{O} 5$ & $2.487(3)$ & $\mathrm{Ce} 1-\mathrm{N} 2^{i i}$ & $2.752(4)$ \\
\hline Ce1-O9 & $2.507(3)$ & $\mathrm{Ce} 1-\mathrm{O} 2^{i v}$ & $2.549(3)$ \\
\hline $\mathrm{Ce} 1-\mathrm{O} 10$ & $2.529(3)$ & $\mathrm{Ce} 1-\mathrm{O}^{v}{ }^{v}$ & $2.516(3)$ \\
\hline Ce1-N1 & $2.681(3)$ & & \\
\hline $\mathrm{O} 1-\mathrm{Ce} 1-\mathrm{O} 5$ & $72.19(9)$ & $\mathrm{O} 9-\mathrm{Ce} 1-\mathrm{N} 2^{i i}$ & 77.53(10) \\
\hline $\mathrm{O} 1-\mathrm{Ce} 1-\mathrm{O} 9$ & $128.95(9)$ & $\mathrm{O} 2^{i v-C e 1-O 9}$ & $68.48(10)$ \\
\hline $\mathrm{O} 1-\mathrm{Ce} 1-\mathrm{O} 10$ & $136.31(9)$ & $\mathrm{O} 6 \mathrm{v}-\mathrm{Ce} 1-\mathrm{O} 9$ & $142.81(10)$ \\
\hline $\mathrm{O} 1-\mathrm{Ce} 1-\mathrm{N} 1$ & $62.30(9)$ & $\mathrm{O} 10-\mathrm{Ce} 1-\mathrm{N} 1$ & $143.40(10)$ \\
\hline $\mathrm{O} 1-\mathrm{Ce} 1-\mathrm{O} 7^{i i}$ & $68.27(9)$ & $\mathrm{O} 7^{i i-} \mathrm{Ce} 1-\mathrm{O} 10$ & $135.15(9)$ \\
\hline $\mathrm{O} 1-\mathrm{Ce} 1-\mathrm{N} 2^{i i}$ & $104.34(9)$ & $\mathrm{O} 10-\mathrm{Ce} 1-\mathrm{N} 2^{i i}$ & $75.39(9)$ \\
\hline
\end{tabular}




\begin{tabular}{|c|c|c|c|}
\hline $\mathrm{O} 1-\mathrm{Ce} 1-\mathrm{O} 2^{i v}$ & $125.58(9)$ & $\mathrm{O} 2^{i v}-\mathrm{Ce} 1-\mathrm{O} 10$ & 71.71(9) \\
\hline $\mathrm{O} 1-\mathrm{Ce} 1-\mathrm{O}^{v}$ & $67.74(9)$ & $\mathrm{O}^{v}-\mathrm{Ce} 1-\mathrm{O} 10$ & $73.19(9)$ \\
\hline O5-Ce1-O9 & $136.14(10)$ & $\mathrm{O} 7^{i i-} \mathrm{Ce} 1-\mathrm{N} 1$ & $77.42(10)$ \\
\hline $\mathrm{O} 5-\mathrm{Ce} 1-\mathrm{O} 10$ & $81.18(9)$ & $\mathrm{N} 1-\mathrm{Ce} 1-\mathrm{N} 2^{i i}$ & 137.53(9) \\
\hline $\mathrm{O} 5-\mathrm{Ce} 1-\mathrm{N} 1$ & $77.08(9)$ & $\mathrm{O} 2^{\mathrm{iv}}-\mathrm{Ce} 1-\mathrm{N} 1$ & $73.09(9)$ \\
\hline $\mathrm{O} 5-\mathrm{Ce} 1-\mathrm{O}^{i i}$ & $139.55(9)$ & $\mathrm{O}^{v}-\mathrm{Ce} 1-\mathrm{N} 1$ & 128.71(9) \\
\hline $\mathrm{O} 5-\mathrm{Ce} 1-\mathrm{N} 2^{i i}$ & $140.55(8)$ & $\mathrm{O} 7^{i i}-\mathrm{Ce} 1-\mathrm{N} 2^{i i}$ & $60.64(9)$ \\
\hline $\mathrm{O} 2^{i v}-\mathrm{Ce} 1-\mathrm{O} 5$ & $68.70(9)$ & $\mathrm{O} 2^{i v}-\mathrm{Ce} 1-\mathrm{O} 7^{i i}$ & $130.94(9)$ \\
\hline $\mathrm{O} 5-\mathrm{Ce} 1-\mathrm{O}^{v}$ & $77.59(9)$ & $\mathrm{O}^{v}-\mathrm{Ce} 1-\mathrm{O} 7^{i i}$ & $94.87(9)$ \\
\hline $\mathrm{O} 9-\mathrm{Ce} 1-\mathrm{O} 10$ & $94.19(9)$ & $\mathrm{O} 2^{i v}-\mathrm{Ce} 1-\mathrm{N} 2^{i i}$ & $129.88(9)$ \\
\hline O9-Ce1-N1 & $81.95(10)$ & $\mathrm{O}^{\mathrm{v}}-\mathrm{Ce} 1-\mathrm{N} 2^{\mathrm{ii}}$ & $65.52(9)$ \\
\hline $\mathrm{O} 7^{i i-} \mathrm{Ce} 1-\mathrm{O} 9$ & $69.28(9)$ & $\mathrm{O} 2^{i v}-\mathrm{Ce} 1-\mathrm{O}^{v}$ & $134.13(8)$ \\
\hline \multicolumn{4}{|c|}{ Symmetry codes: (i) $1+x,+y,+z$; (ii) $2-x, 1-y,-z$; (iii) $+x,-1+y,+z$; (iv) $-1+x,+y,+z$; (v) $+x, 1+y,+z$} \\
\hline \multicolumn{4}{|l|}{3} \\
\hline $\mathrm{Ce} 1-\mathrm{O} 1$ & $2.599(5)$ & $\mathrm{Ce} 2-\mathrm{O} 2 \mathrm{~W}$ & $2.609(10)$ \\
\hline Ce1-O1W & $2.643(12)$ & $\mathrm{Ce} 2-\mathrm{O} 3$ & $2.452(6)$ \\
\hline $\mathrm{Ce} 1-\mathrm{O} 11$ & $2.485(12)$ & $\mathrm{Ce} 2-\mathrm{O} 7$ & $2.425(6)$ \\
\hline Ce1-N1 & $2.698(8)$ & $\mathrm{Ce} 2-\mathrm{O} 1^{i i}$ & $2.668(5)$ \\
\hline $\mathrm{Ce} 1-\mathrm{O}^{i}$ & $2.475(6)$ & $\mathrm{Ce} 2-\mathrm{O} 2^{i i}$ & $2.638(6)$ \\
\hline $\mathrm{Ce} 1-\mathrm{N} 2^{i}$ & $2.685(7)$ & $\mathrm{Ce} 2-\mathrm{O}^{x}$ & $2.668(5)$ \\
\hline $\mathrm{Ce} 1-\mathrm{O} 4^{i i i}$ & $2.436(6)$ & $\mathrm{Ce} 2-\mathrm{O} 2^{x}$ & $2.638(6)$ \\
\hline $\mathrm{Ce} 1-\mathrm{O}^{i i i}$ & $2.544(9)$ & $\mathrm{Ce} 2-\mathrm{O}^{x i}$ & $2.452(6)$ \\
\hline
\end{tabular}




\begin{tabular}{|c|c|c|c|}
\hline $\mathrm{Ce} 1-\mathrm{O}^{\text {viii }}$ & $2.434(5)$ & $\mathrm{Ce} 2-\mathrm{O}^{x i}$ & $2.425(6)$ \\
\hline $\mathrm{O} 1-\mathrm{Ce} 1-\mathrm{O} 1 \mathrm{~W}$ & $135.3(3)$ & $\mathrm{O} 1-\mathrm{Ce} 1-\mathrm{O}^{i i i}$ & $66.0(2)$ \\
\hline $\mathrm{O} 5^{i-} \mathrm{Ce} 1-\mathrm{O} 8^{i i i}$ & $125.9(3)$ & $\mathrm{O} 4^{i i i}-\mathrm{Ce} 1-\mathrm{O} 6^{\text {viii }}$ & $147.1(2)$ \\
\hline $\mathrm{O} 1-\mathrm{Ce} 1-\mathrm{O} 11$ & $110.0(3)$ & $\mathrm{O} 1-\mathrm{Ce} 1-\mathrm{O}^{\text {viii }}$ & $130.36(18)$ \\
\hline $\mathrm{O} 5^{i}-\mathrm{Ce} 1-\mathrm{O}^{v i i i}$ & $68.60(19)$ & $\mathrm{O}^{\text {viii }}-\mathrm{Ce} 1-\mathrm{O} 8^{i i i}$ & $72.3(3)$ \\
\hline $\mathrm{O} 1-\mathrm{Ce} 1-\mathrm{N} 1$ & $60.1(2)$ & $\mathrm{O} 1 \mathrm{~W}-\mathrm{Ce} 1-\mathrm{O} 11$ & $87.9(4)$ \\
\hline $\mathrm{O} 4^{i i i-} \mathrm{Ce} 1-\mathrm{N} 2^{i}$ & $75.4(2)$ & $\mathrm{O} 2 \mathrm{~W}-\mathrm{Ce} 2-\mathrm{O} 3$ & $73.6(2)$ \\
\hline $\mathrm{O} 1-\mathrm{Ce} 1-\mathrm{O} 5^{i}$ & $117.20(19)$ & $\mathrm{O} 1 \mathrm{~W}-\mathrm{Ce} 1-\mathrm{N} 1$ & $133.0(3)$ \\
\hline $\mathrm{O} 8^{i i i-} \mathrm{Ce} 1-\mathrm{N} 2^{i}$ & $132.1(3)$ & $\mathrm{O} 2 \mathrm{~W}-\mathrm{Ce} 2-\mathrm{O} 7$ & $67.9(2)$ \\
\hline $\mathrm{O} 1-\mathrm{Ce} 1-\mathrm{N} 2^{i}$ & $69.46(19)$ & $\mathrm{O} 1 \mathrm{~W}-\mathrm{Ce} 1-\mathrm{O}^{i i i}$ & $61.3(3)$ \\
\hline $\mathrm{O} 6^{v i i i}-\mathrm{Ce} 1-\mathrm{N} 2^{i}$ & $130.9(2)$ & $\mathrm{O} 1^{i i}-\mathrm{Ce} 2-\mathrm{O} 2 \mathrm{~W}$ & $130.95(12)$ \\
\hline $\mathrm{O} 1-\mathrm{Ce} 1-\mathrm{O} 4^{i i i}$ & $72.74(19)$ & $\mathrm{O} 1 \mathrm{~W}-\mathrm{Ce} 1-\mathrm{N} 2^{i}$ & $72.3(3)$ \\
\hline $\mathrm{O} 4^{i i i-} \mathrm{Ce} 1-\mathrm{O} 8^{i i i}$ & $106.4(3)$ & $\mathrm{O} 2^{i i}-\mathrm{Ce} 2-\mathrm{O} 2 \mathrm{~W}$ & $137.8(2)$ \\
\hline $\mathrm{O} 1 \mathrm{~W}-\mathrm{Ce} 1-\mathrm{O} 4^{i i i}$ & $75.9(3)$ & $\mathrm{O} 11-\mathrm{Ce} 1-\mathrm{N} 1$ & $133.4(3)$ \\
\hline $\mathrm{O} 1^{x}-\mathrm{Ce} 2-\mathrm{O} 2 \mathrm{~W}$ & $130.95(12)$ & $\mathrm{O}^{i}-\mathrm{Ce} 1-\mathrm{O} 11$ & $132.7(3)$ \\
\hline $\mathrm{O} 1 \mathrm{~W}-\mathrm{Ce} 1-\mathrm{O} 8^{i i i}$ & $155.5(3)$ & $\mathrm{O} 11-\mathrm{Ce} 1-\mathrm{N} 2^{i}$ & $143.7(3)$ \\
\hline $\mathrm{O} 2^{x}-\mathrm{Ce} 2-\mathrm{O} 2 \mathrm{~W}$ & $137.8(2)$ & $\mathrm{O} 4^{i i i-}-\mathrm{Ce} 1-\mathrm{O} 11$ & $70.3(3)$ \\
\hline $\mathrm{O} 1 \mathrm{~W}-\mathrm{Ce} 1-\mathrm{O}^{v i i i}$ & $92.5(3)$ & $\mathrm{O} 8^{i i i}-\mathrm{Ce} 1-\mathrm{O} 11$ & $70.7(3)$ \\
\hline $\mathrm{O} 2 \mathrm{~W}-\mathrm{Ce} 2-\mathrm{O} 3^{x i}$ & $73.6(2)$ & $\mathrm{O} 6^{v i i i}-\mathrm{Ce} 1-\mathrm{O} 11$ & $78.7(3)$ \\
\hline $\mathrm{O} 5^{i}-\mathrm{Ce} 1-\mathrm{N} 1$ & $72.6(3)$ & $\mathrm{O} 4^{i i i}-\mathrm{Ce} 1-\mathrm{O}^{i}$ & $126.8(2)$ \\
\hline $\mathrm{N} 1-\mathrm{Ce} 1-\mathrm{N} 2^{i}$ & $79.4(3)$ & $\mathrm{O} 7-\mathrm{Ce} 2-\mathrm{O} 7^{x i}$ & $135.9(2)$ \\
\hline $\mathrm{O} 4^{i i i}-\mathrm{Ce} 1-\mathrm{N} 1$ & $131.8(2)$ & $\mathrm{O} 1^{i i}-\mathrm{Ce} 2-\mathrm{O} 2^{i i}$ & $49.14(17)$ \\
\hline
\end{tabular}




\begin{tabular}{|c|c|c|c|}
\hline $\mathrm{O}^{i i i}-\mathrm{Ce} 1-\mathrm{N} 1$ & $63.9(3)$ & $\mathrm{O} 2 \mathrm{~W}-\mathrm{Ce} 2-\mathrm{O} 7^{x i}$ & $67.9(2)$ \\
\hline $\mathrm{O}^{\text {viii }}-\mathrm{Ce} 1-\mathrm{N} 1$ & $78.3(2)$ & $\mathrm{O} 3-\mathrm{Ce} 2-\mathrm{O} 7$ & $86.0(2)$ \\
\hline $\mathrm{O} 5^{i}-\mathrm{Ce} 1-\mathrm{N} 2^{i}$ & $63.1(2)$ & $\mathrm{O} 1^{i i}-\mathrm{Ce} 2-\mathrm{O} 3$ & $81.33(18)$ \\
\hline $\mathrm{O} 2^{i i}-\mathrm{Ce} 2-\mathrm{O} 3$ & $130.43(18)$ & $\mathrm{O} 2^{x}-\mathrm{Ce} 2-\mathrm{O} 7$ & $138.68(19)$ \\
\hline $\mathrm{O} 1^{x}-\mathrm{Ce} 2-\mathrm{O} 3$ & $121.45(19)$ & $\mathrm{O} 3^{x i}-\mathrm{Ce} 2-\mathrm{O} 7$ & $81.8(2)$ \\
\hline $\mathrm{O} 2^{x}-\mathrm{Ce} 2-\mathrm{O} 3$ & $76.8(2)$ & $\mathrm{O} 1^{i}-\mathrm{Ce} 2-\mathrm{O} 1^{x}$ & $98.09(17)$ \\
\hline $\mathrm{O} 3-\mathrm{Ce} 2-\mathrm{O}^{x i}$ & $147.1(2)$ & $\mathrm{O} 1^{i}-\mathrm{Ce} 2-\mathrm{O} 2^{x}$ & $71.54(17)$ \\
\hline $\mathrm{O} 3-\mathrm{Ce} 2-\mathrm{O} 7^{x i}$ & $81.8(2)$ & $\mathrm{O} 1^{i}-\mathrm{Ce} 2-\mathrm{O} 3^{x i}$ & $121.45(19)$ \\
\hline $\mathrm{O} 1^{i i}-\mathrm{Ce} 2-\mathrm{O} 7$ & $68.9(2)$ & $\mathrm{O} 1^{i}-\mathrm{Ce} 2-\mathrm{O} 7^{x i}$ & $148.5(2)$ \\
\hline $\mathrm{O} 2^{i i}-\mathrm{Ce} 2-\mathrm{O} 7$ & $78.8(2)$ & $\mathrm{O} 1^{x}-\mathrm{Ce} 2-\mathrm{O} 2^{i i}$ & $71.54(17)$ \\
\hline $\mathrm{O}^{x}-\mathrm{Ce} 2-\mathrm{O} 7$ & $148.5(2)$ & $\mathrm{O} 2^{i i}-\mathrm{Ce} 2-\mathrm{O} 2^{x}$ & $84.43(18)$ \\
\hline $\mathrm{O} 2^{i i}-\mathrm{Ce} 2-\mathrm{O} 3^{x i}$ & $76.8(2)$ & $\mathrm{O} 2^{x}-\mathrm{Ce} 2-\mathrm{O} 3^{x i}$ & $130.43(18)$ \\
\hline $\mathrm{O} 2^{i i}-\mathrm{Ce} 2-\mathrm{O} 7^{x i}$ & $138.68(19)$ & $\mathrm{O} 2^{x}-\mathrm{Ce} 2-\mathrm{O} 7^{x i}$ & $78.8(2)$ \\
\hline $\mathrm{O} 1^{x}-\mathrm{Ce} 2-\mathrm{O} 2^{x}$ & $49.14(17)$ & $\mathrm{O} 3^{x i}-\mathrm{Ce} 2-\mathrm{O} 7^{x i}$ & $86.0(2)$ \\
\hline $\mathrm{O} 1^{x}-\mathrm{Ce} 2-\mathrm{O} 3^{x i}$ & $81.33(18)$ & $\mathrm{Ce} 1-\mathrm{O} 1-\mathrm{Ce} 2^{i i i}$ & $127.3(2)$ \\
\hline $\mathrm{O} 1^{x}-\mathrm{Ce} 2-\mathrm{O} 7^{x i}$ & $68.9(2)$ & & \\
\hline
\end{tabular}

Symmetry codes: (i) $-1+x, 1+y, z$; (ii) $x,-1+y, z$; (iii) $x, 1+y, z$; (viii) $-1 / 2+x, 3 / 2-y, 5 / 4-z$; (x) $1+y, x, 1-z ;$ (xi) $y, x, 1-z$ 
Table S2. Hydrogen bonds geometry $\left(\AA,{ }^{\circ}\right)$ for $\mathbf{1}, \mathbf{2}$ and $\mathbf{3}$

\begin{tabular}{|c|c|c|c|c|}
\hline $\mathbf{D}-\mathbf{H} \cdots \mathbf{A}$ & $\mathbf{D}-\mathbf{H}$ & $\mathbf{H} \cdots \mathbf{A}$ & $\mathbf{D} \cdots A$ & $\mathbf{D}-\mathbf{H} \cdots \mathbf{A}$ \\
\hline 1 & & & & \\
\hline $\mathrm{N} 1-\mathrm{H} 1 \cdots \mathrm{O} 1$ & $0.88(3)$ & $2.29(5)$ & $2.661(6)$ & $106(5)$ \\
\hline $\mathrm{N} 1-\mathrm{H} 1 \cdots \mathrm{O} 12$ & $0.88(3)$ & $2.10(4)$ & $2.957(6)$ & $163(5)$ \\
\hline O9-H9A $\cdots$ O4 & $0.84(4)$ & $1.94(4)$ & $2.784(5)$ & $179(7)$ \\
\hline O9-H9B $\cdots \mathrm{O} 7$ & $0.84(3)$ & $1.96(5)$ & $2.752(5)$ & $156(6)$ \\
\hline $\mathrm{O} 10-\mathrm{H} 10 \mathrm{~A} \cdots \mathrm{O} 5$ & $0.84(4)$ & $2.29(5)$ & $2.992(5)$ & $142(5)$ \\
\hline O10-H10A $\cdots$ O9 & $0.84(4)$ & $2.32(4)$ & $2.943(6)$ & $132(5)$ \\
\hline O10-H10B $\cdots$ O6 & $0.84(2)$ & $1.92(2)$ & $2.752(6)$ & $176(6)$ \\
\hline $\mathrm{O} 11-\mathrm{H} 11 \mathrm{~A} \cdots \mathrm{O} 4$ & $0.84(4)$ & $2.04(3)$ & $2.876(6)$ & $170(8)$ \\
\hline $\mathrm{O} 11-\mathrm{H} 11 \mathrm{~B} \cdots \mathrm{O} 3$ & $0.84(5)$ & $1.88(4)$ & $2.713(6)$ & $168(8)$ \\
\hline $\mathrm{O} 12-\mathrm{H} 12 \mathrm{~A} \cdots \mathrm{O} 2$ & $0.84(4)$ & $1.88(4)$ & $2.672(6)$ & $158(7)$ \\
\hline $\mathrm{O} 12-\mathrm{H} 12 \mathrm{~B} \cdots \mathrm{O} 6$ & $0.85(5)$ & $1.89(5)$ & $2.727(5)$ & $171(7)$ \\
\hline
\end{tabular}

\begin{tabular}{ccccc}
\hline $\mathbf{D}-\mathbf{H} \cdots \mathbf{A}$ & $\mathbf{D}-\mathbf{H}$ & $\mathbf{H} \cdots \mathbf{A}$ & $\mathbf{D} \cdots \mathbf{A}$ & $\mathbf{D}-\mathbf{H} \cdots \mathbf{A}$ \\
\hline $\mathbf{2}$ & & & & \\
\hline O4-H4 $\cdots \mathrm{O} 7$ & 0.84 & 1.74 & $2.570(4)$ & 168 \\
O9-H9A $\cdots$ O8 & $0.83(3)$ & $1.91(3)$ & $2.731(4)$ & $169(4)$ \\
O9-H9B $\cdots \mathrm{O} 3$ & $0.83(3)$ & $2.14(3)$ & $2.910(4)$ & $153(4)$ \\
O10-H10A $\cdots \mathrm{O} 1$ & $0.84(4)$ & $1.87(4)$ & $2.683(4)$ & $161(4)$
\end{tabular}



O10-H10B $\cdots$ O5
$0.84(2)$
$1.96(3)$
$2.729(4)$
$152(4)$

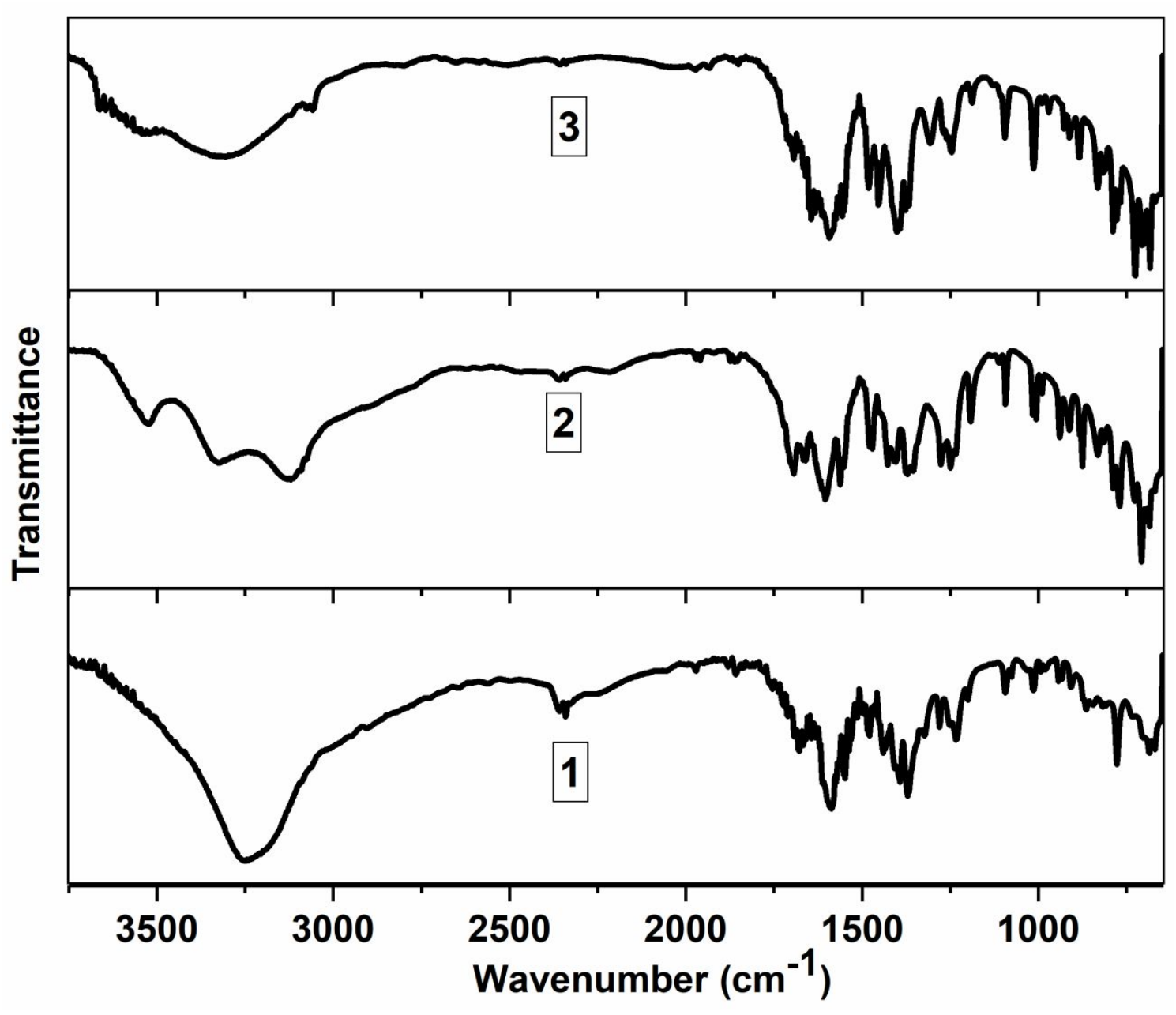

Fig. S1 DRIFT spectra of compounds 1, 2 and $\mathbf{3}$. 

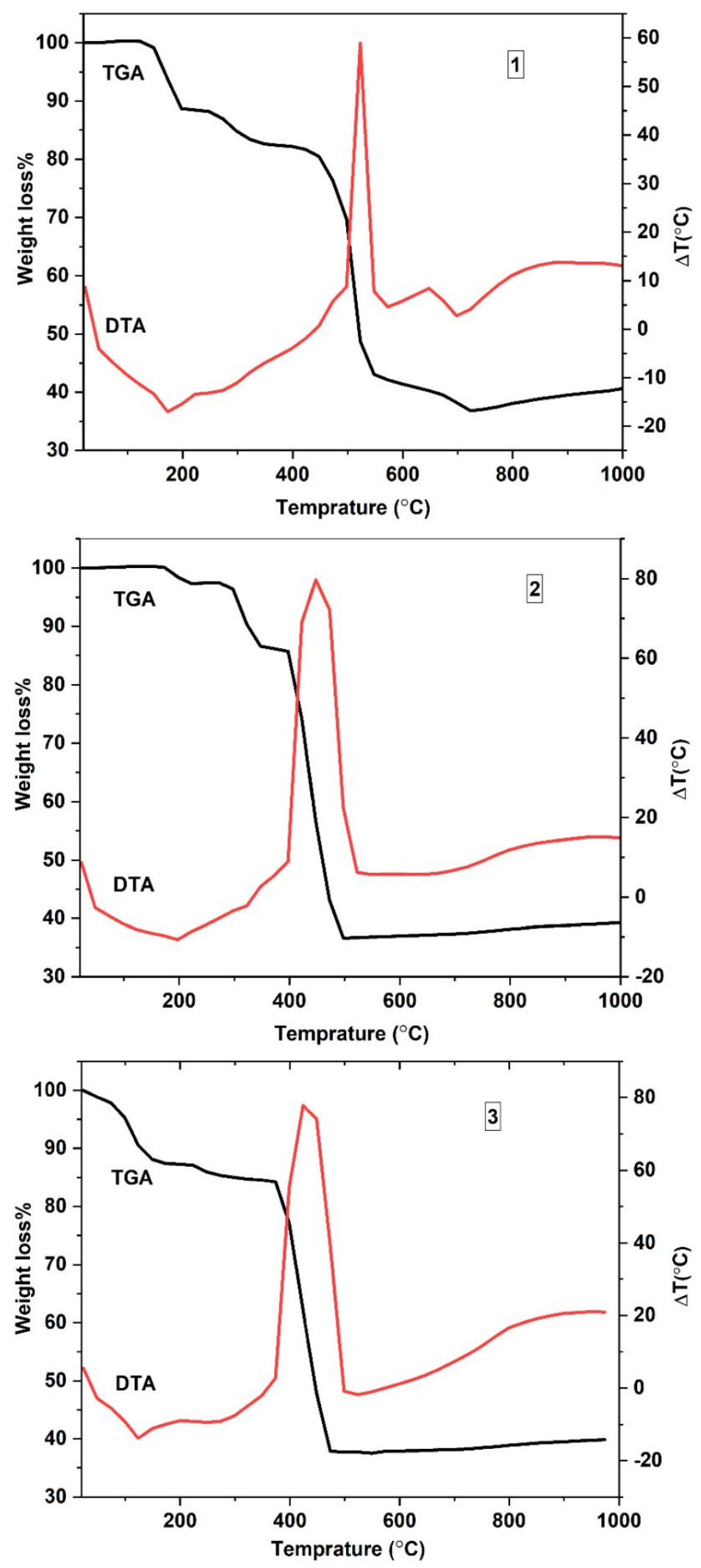

Fig. S2 TGA and DTA curves of compounds 1, 2 and $\mathbf{3 .}$ 

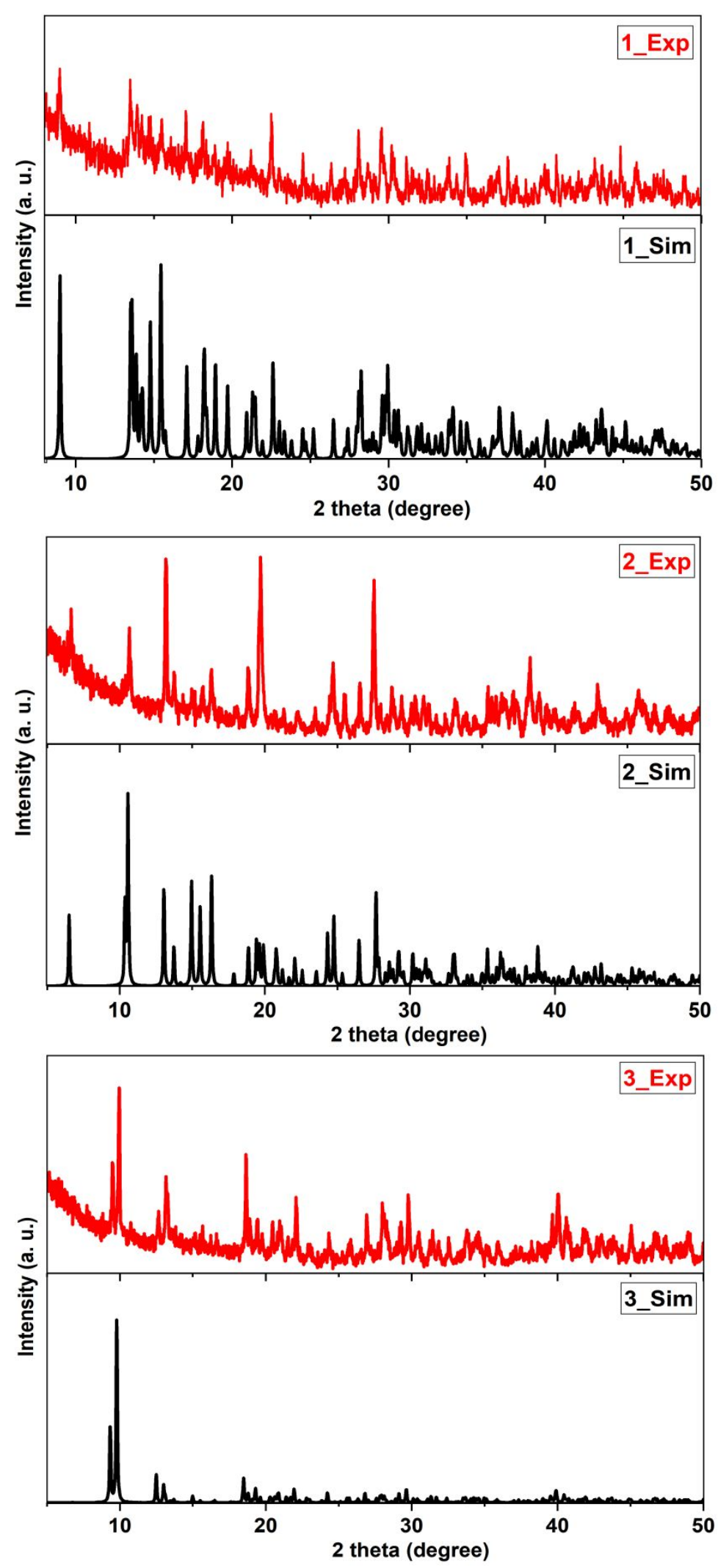

Fig. S3 XRD patterns of compounds 1, 2 and 3 (Simulated and Experimental). 


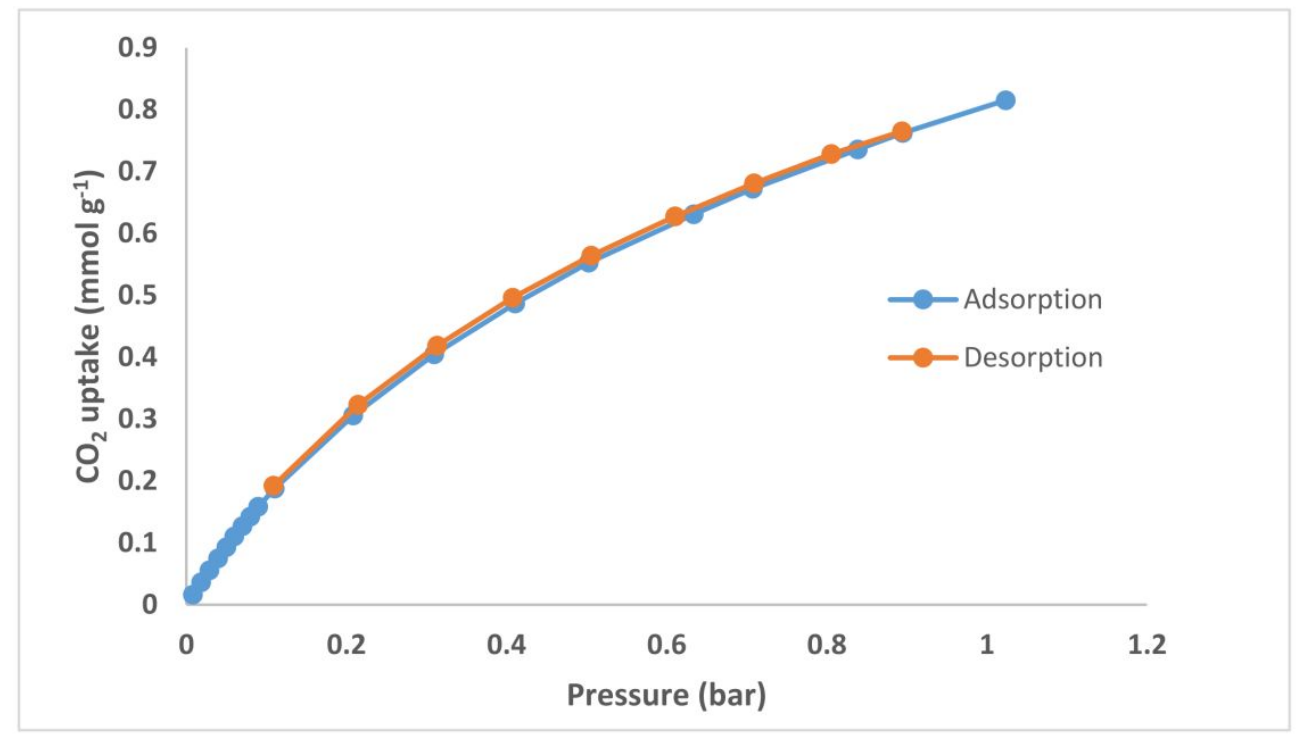

Fig. S4 $\mathrm{CO}_{2}$ adsorption isotherm of $\mathbf{3}$ measured at $298 \mathrm{~K}$.

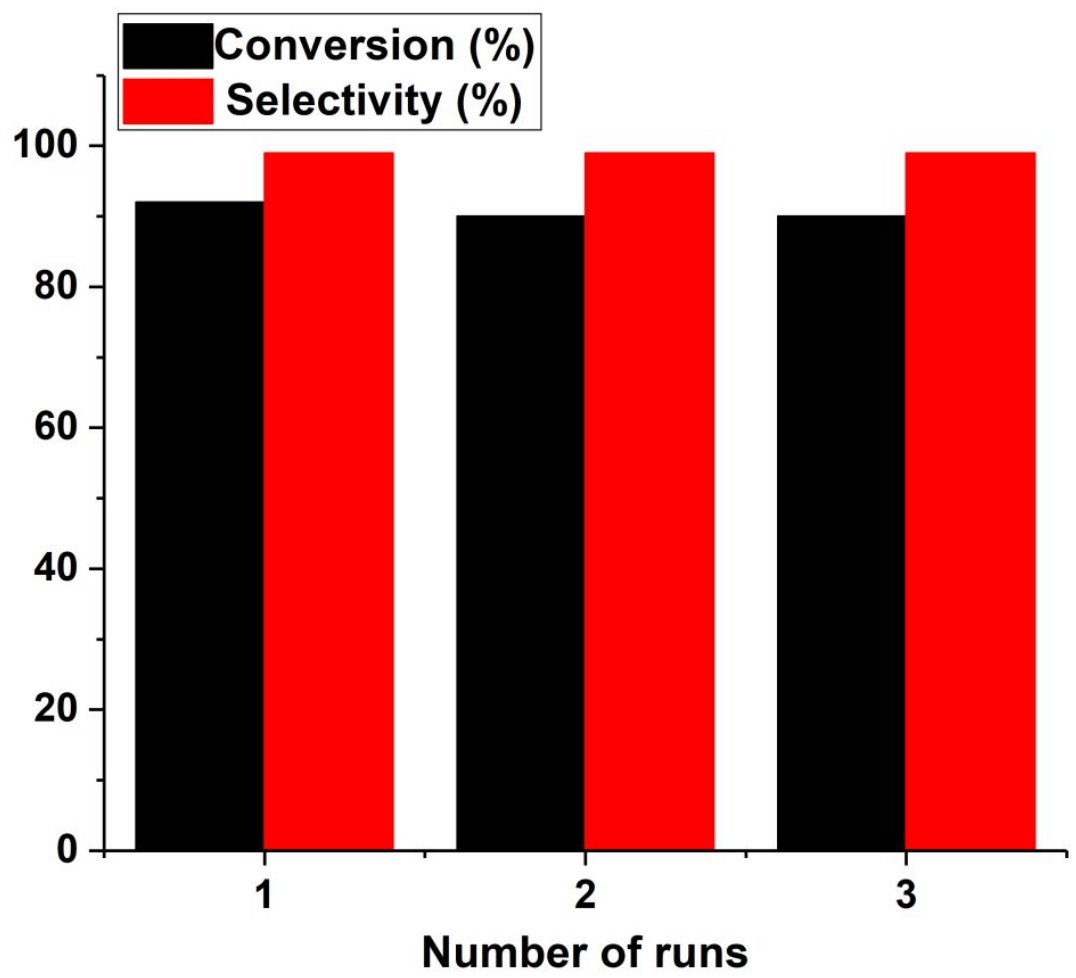

Fig. S5 Recyclability of the compound 3. 


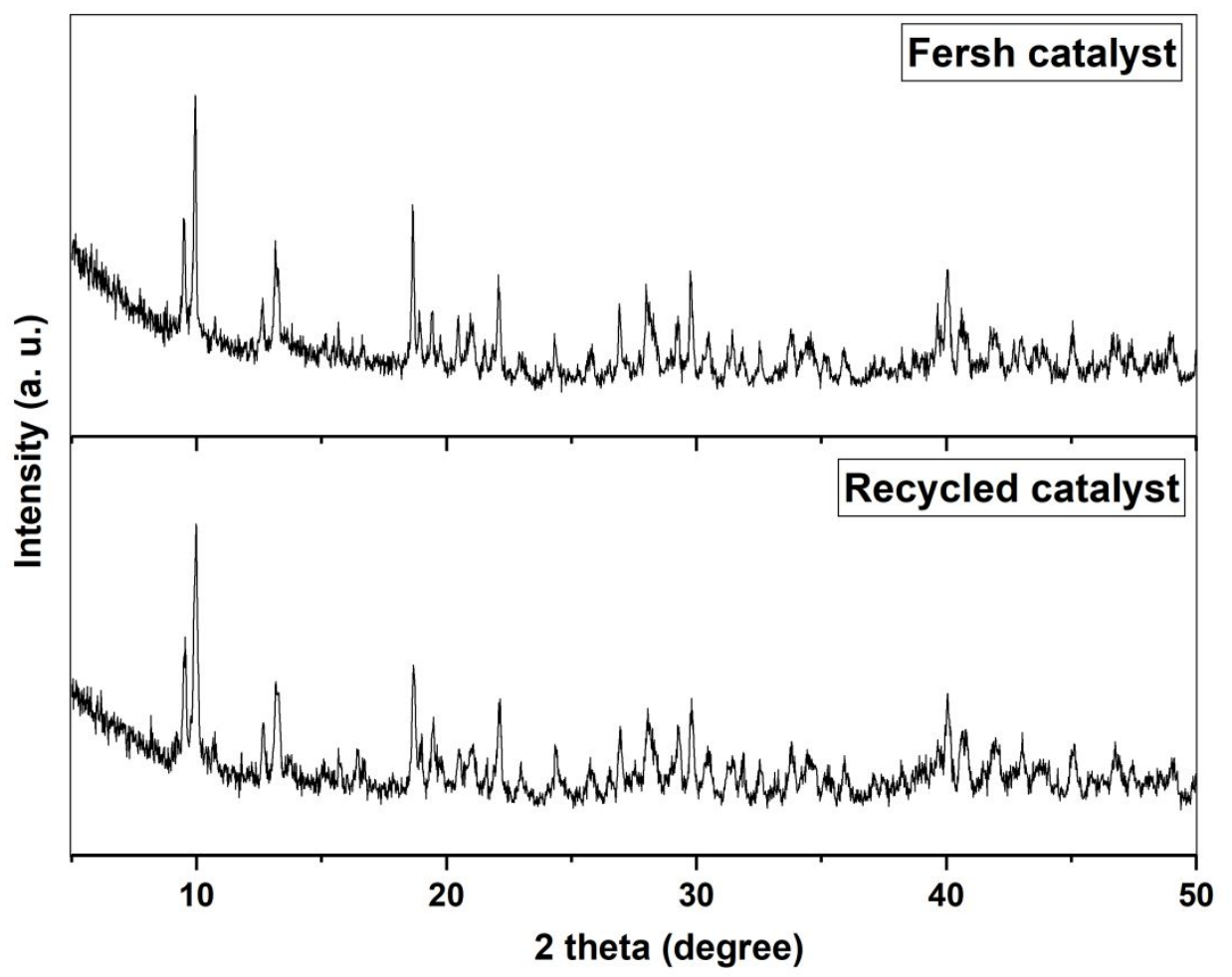

Fig. S6 XRD patterns of the compound 3 before and after the catalytic reaction. 


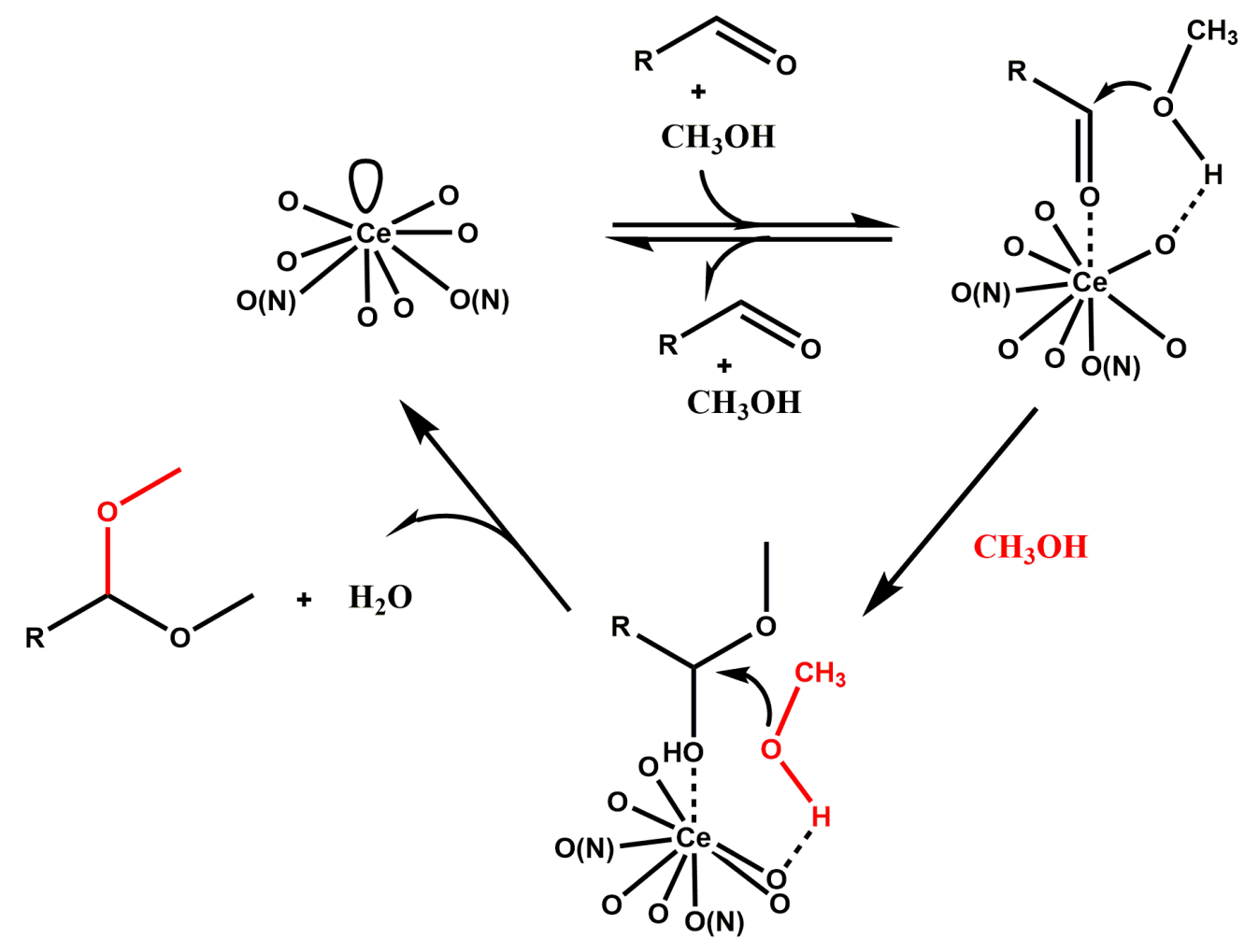

Fig. S7. The proposed reaction mechanism for the Ce MOF-catalyzed acetalization of benzaldehyde with methanol.

\section{GC-MS analysis}

The data of the GC-MS analyses are provided below. In all chromatogram, the peaks are assigned to the related materials. The peak in the retention time of $10.40 \mathrm{~min}$ is related to the dodecane. Please see the spectra in the next pages. 


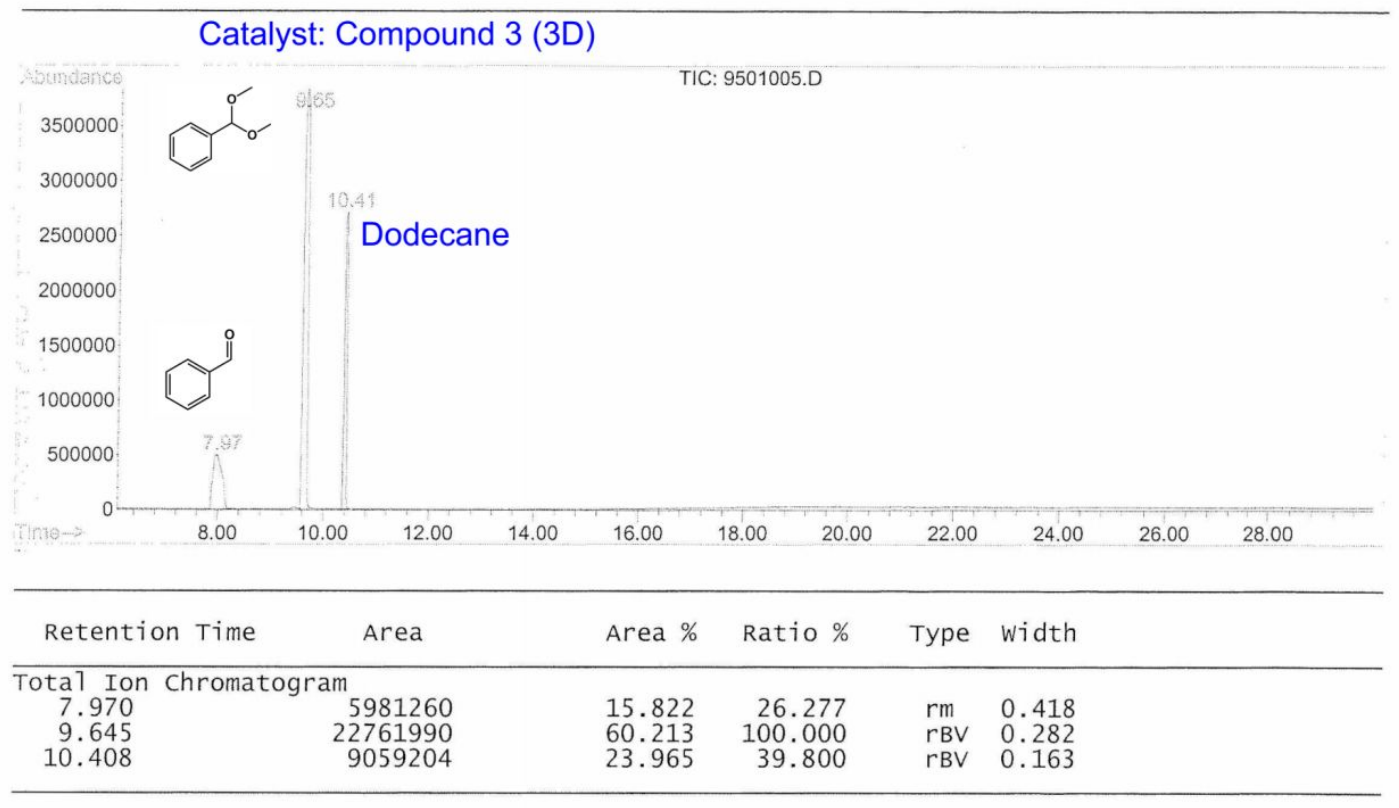




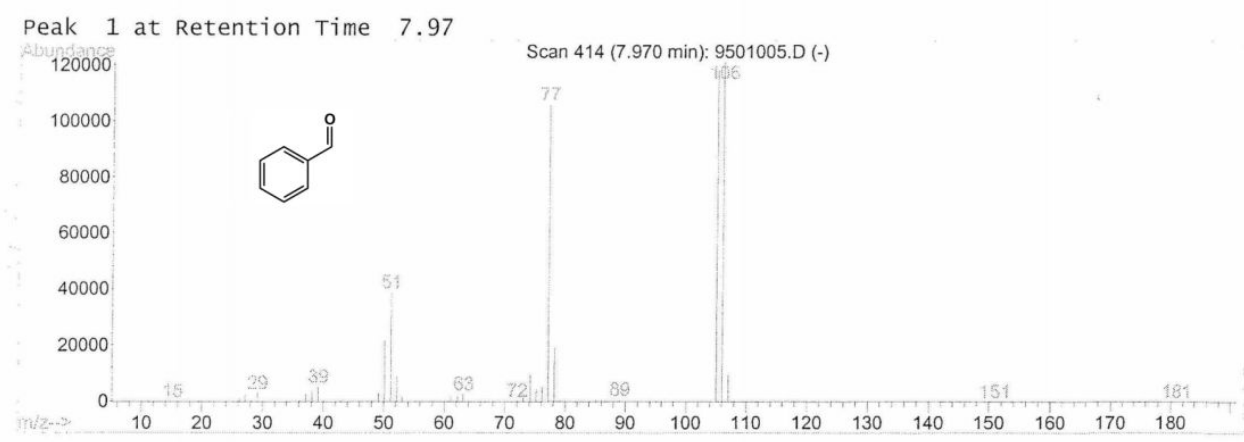

Peak 2 at Retention Time 9.65

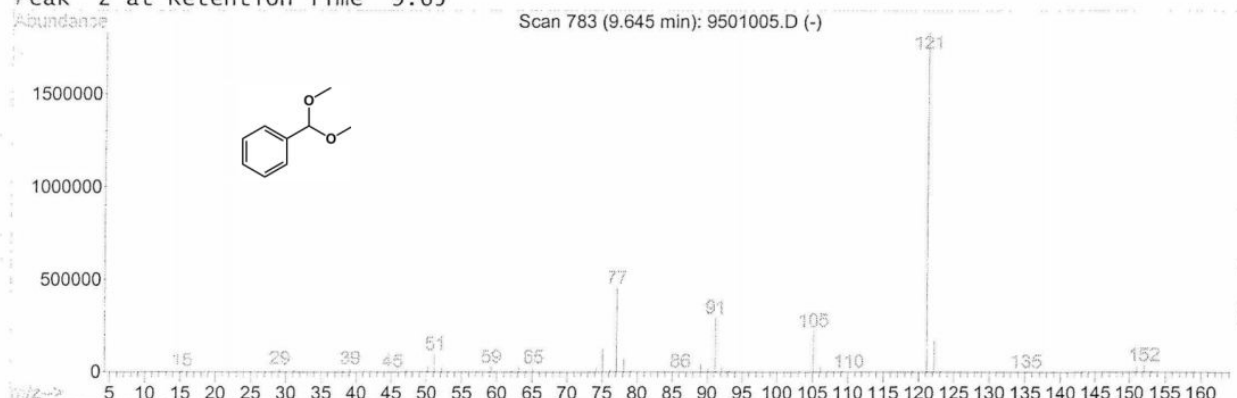

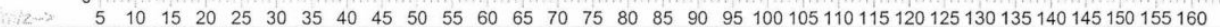

Peak 3 at Retention Time 10.41

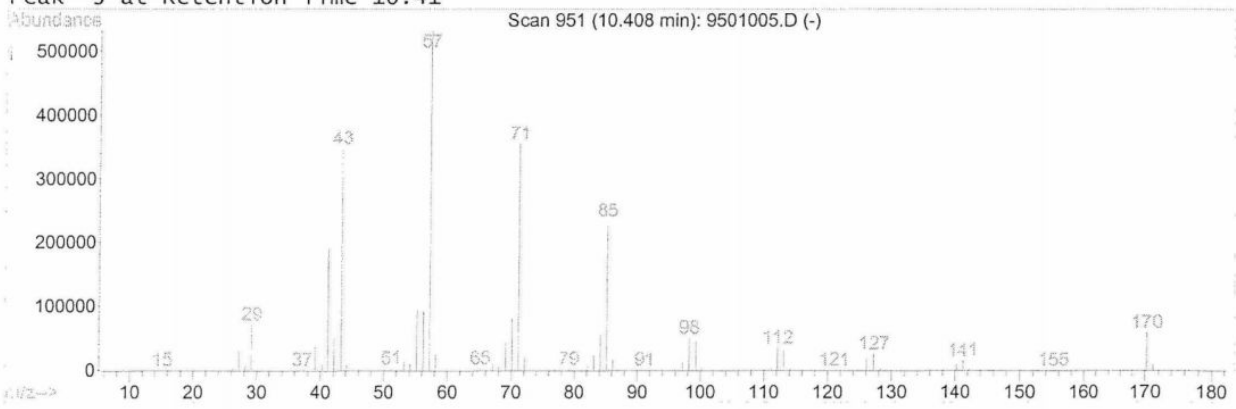

D: \DATA \19-09-17\9501005.D 


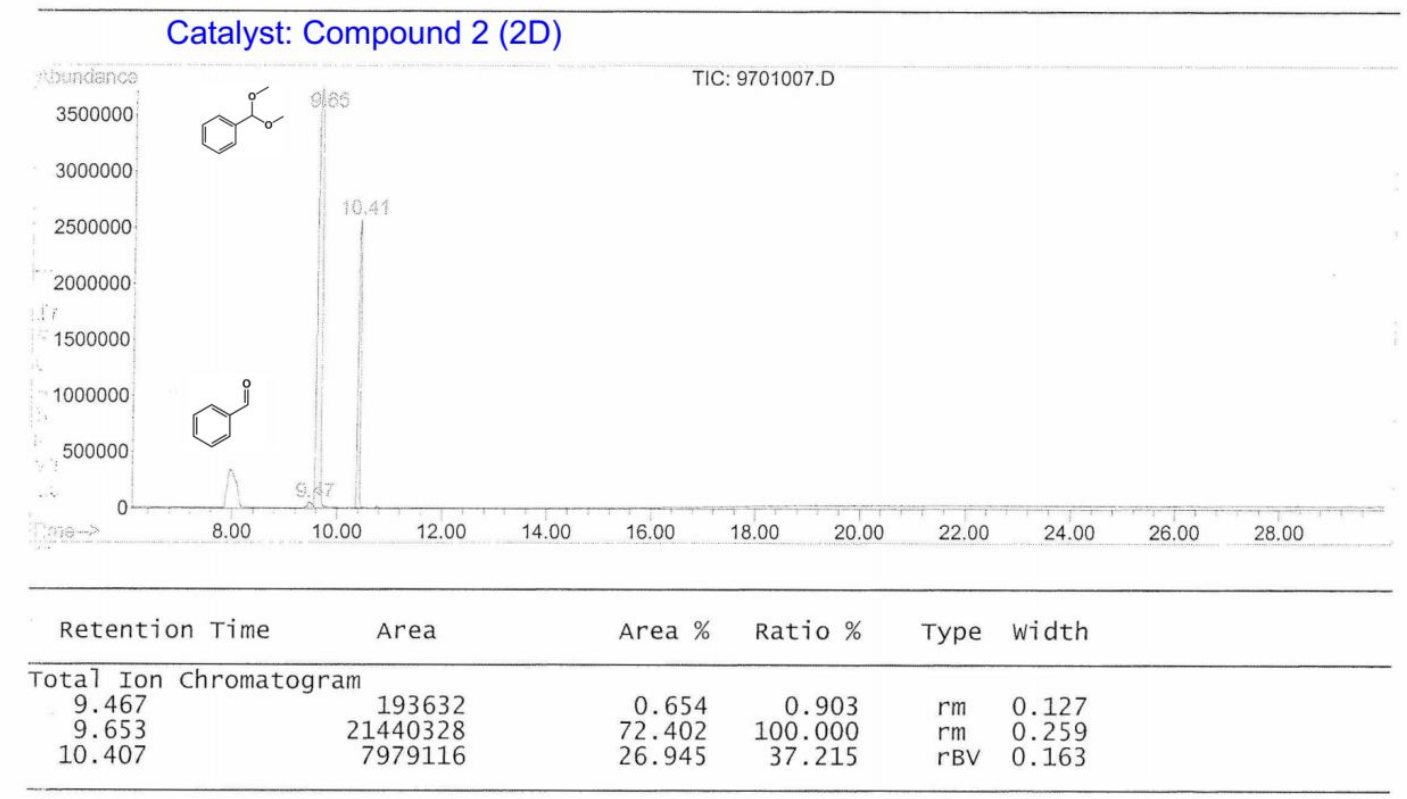




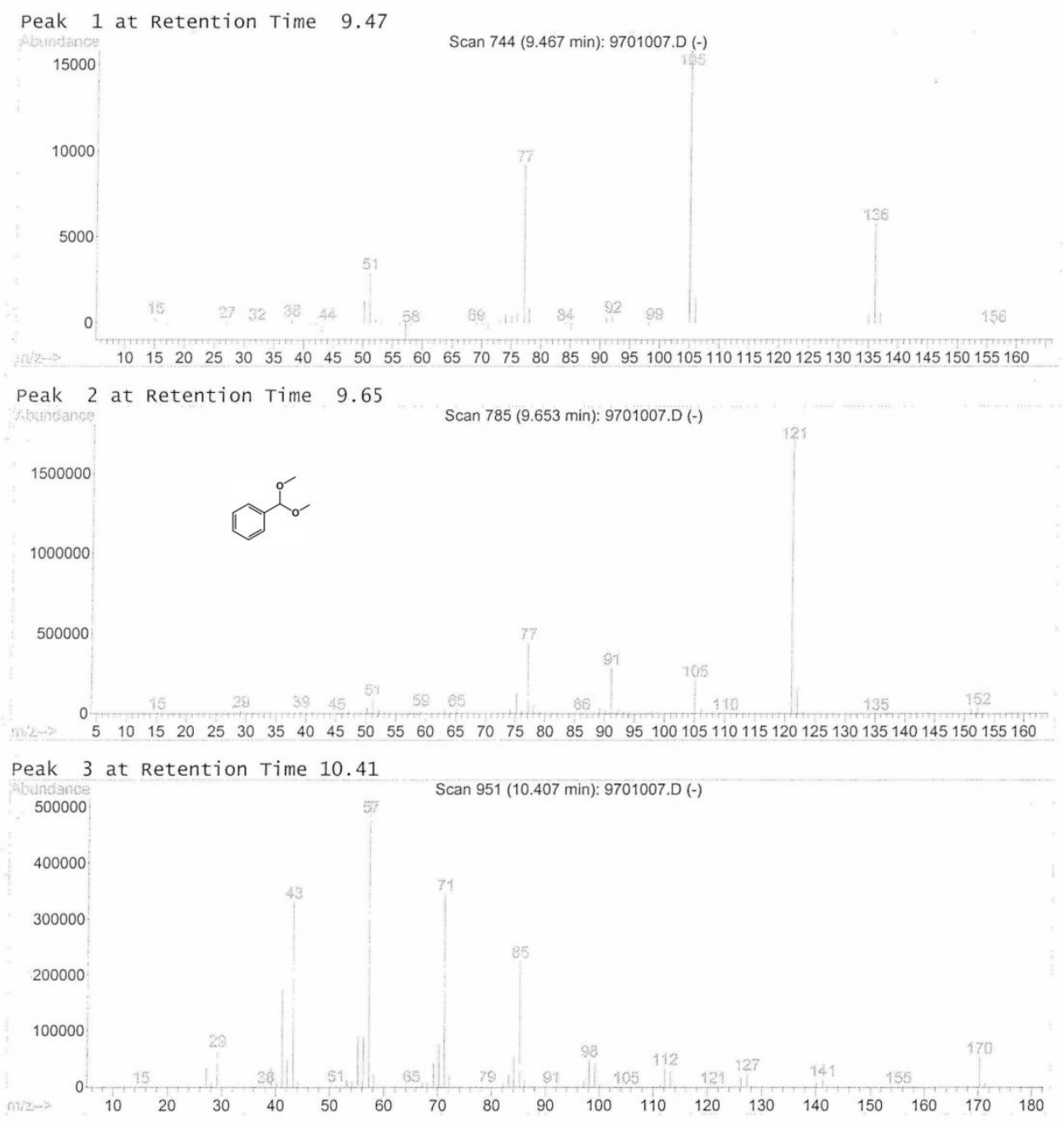




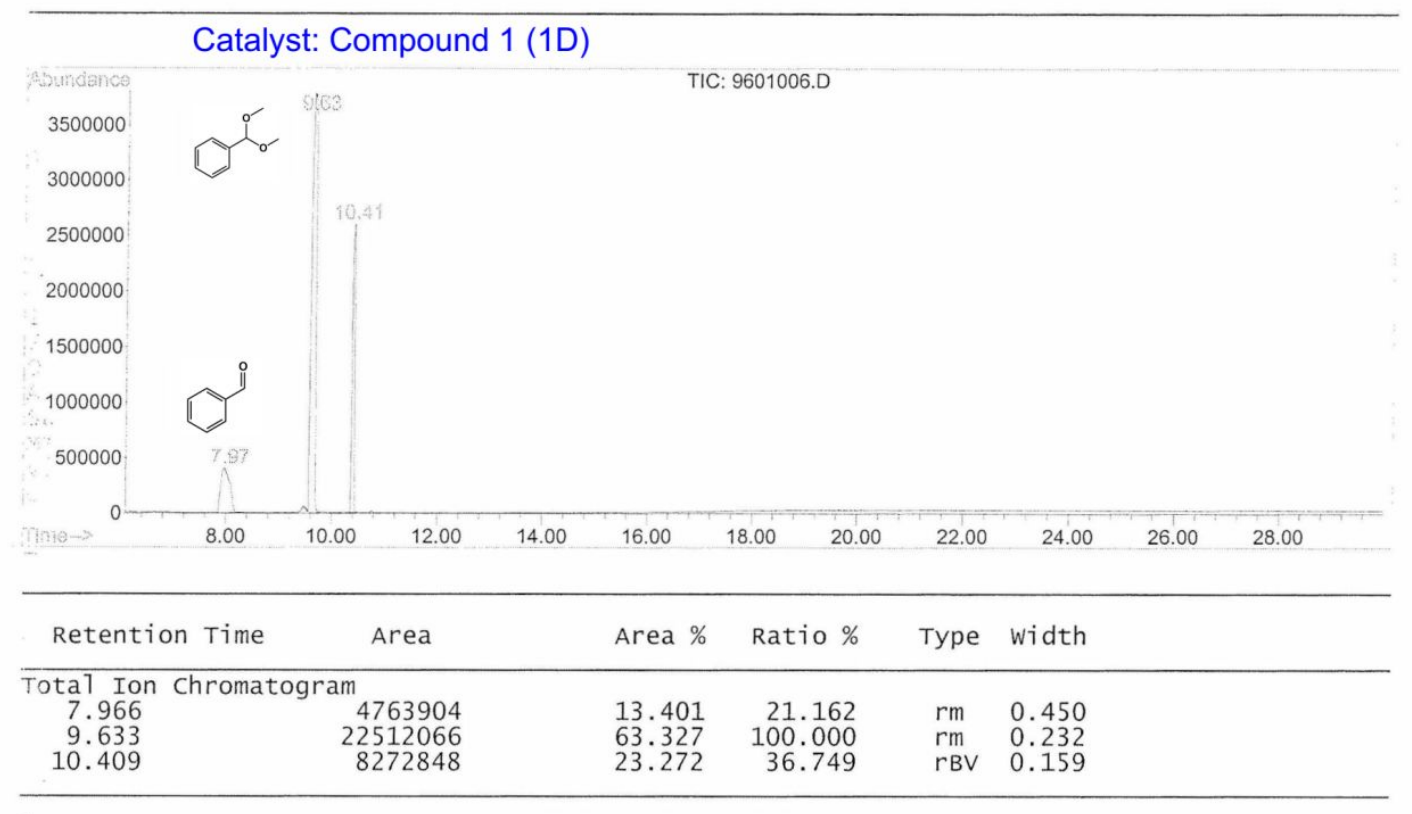

D: \DATA \19-09-17\9601006.D

Thu Sep 19 10:13:19 2019

Page 1 


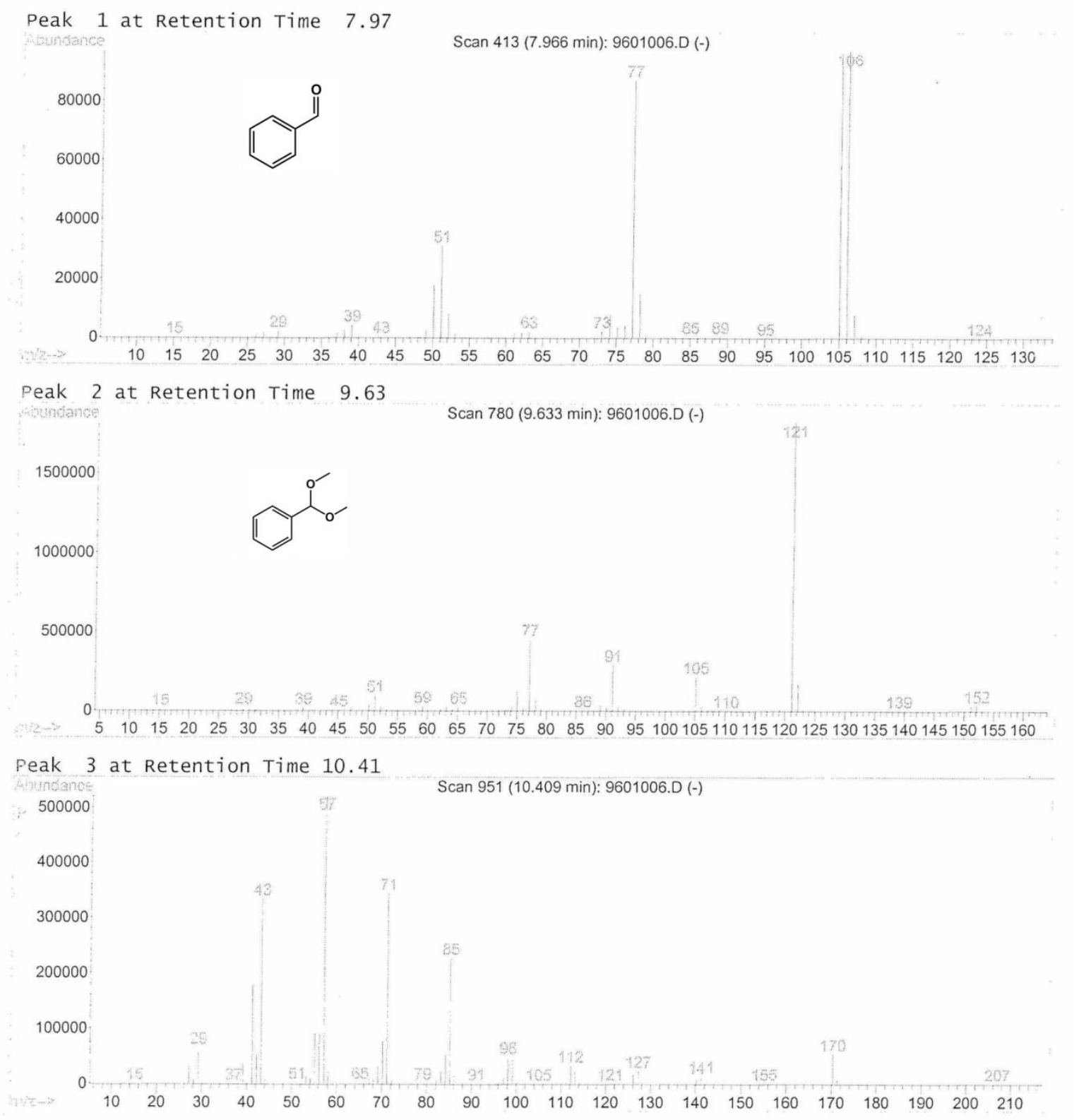




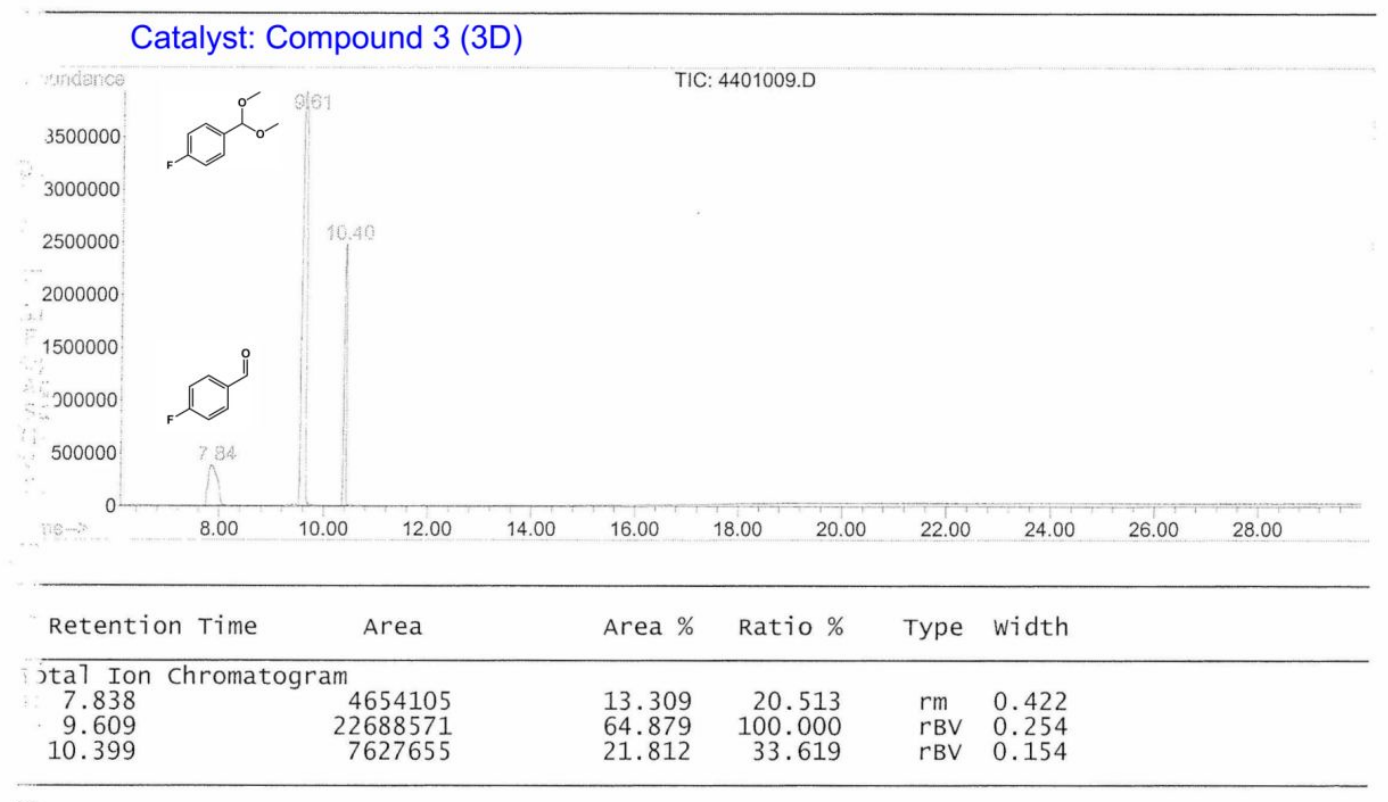




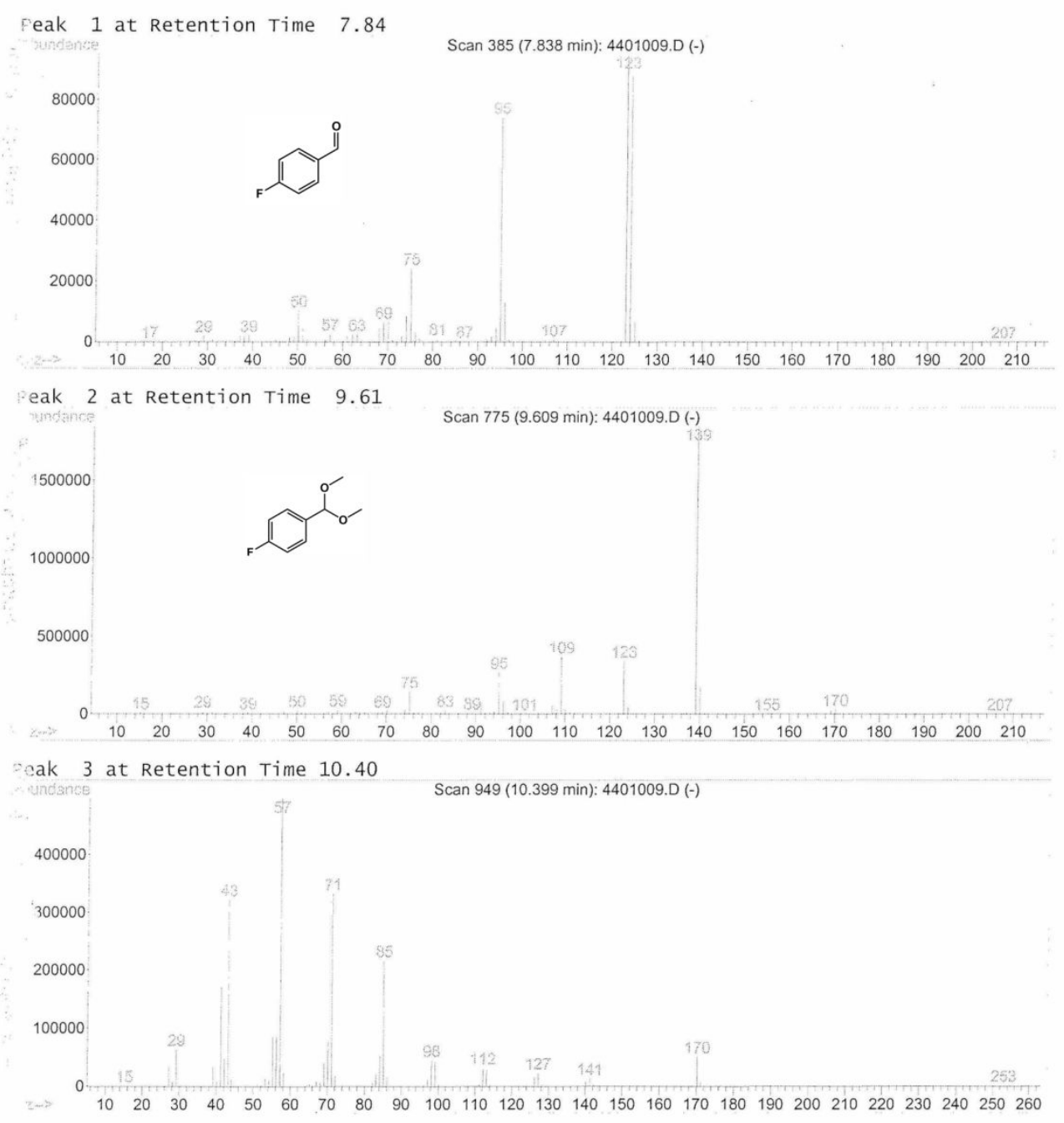

U: \DATA $19-09-11 \backslash 4401009 . D$ 


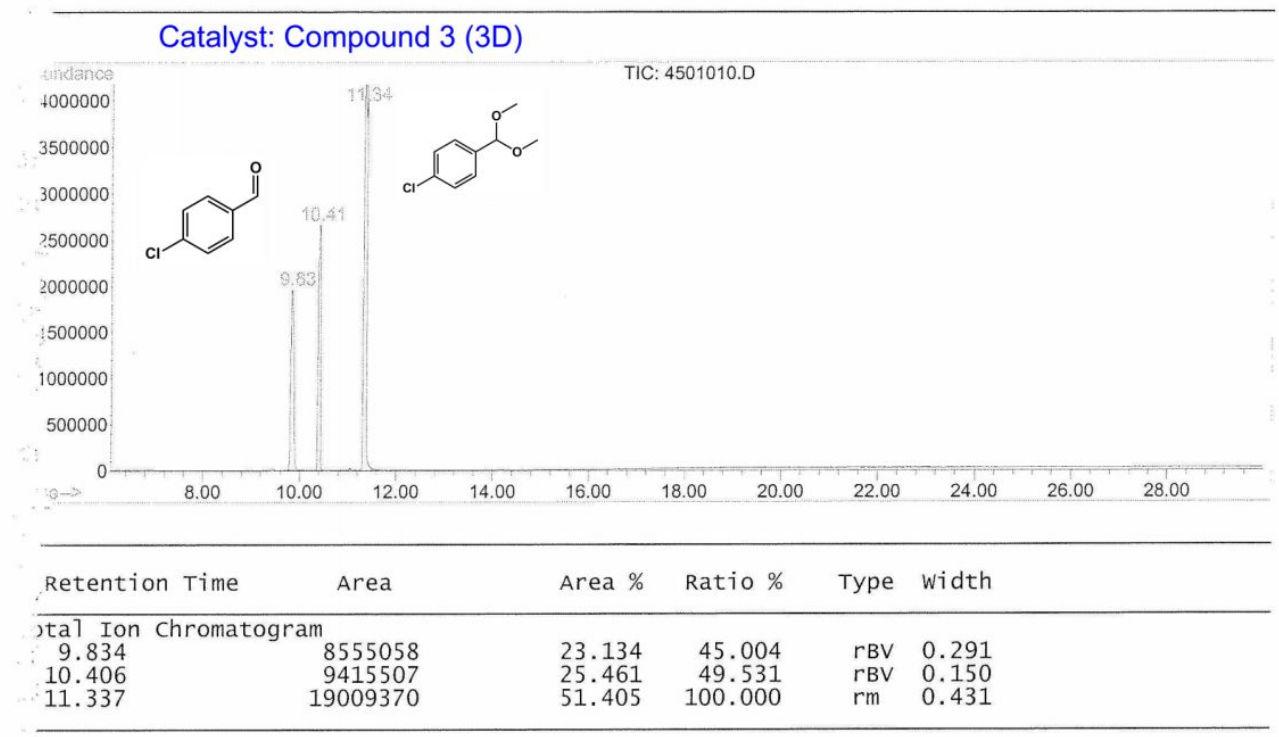




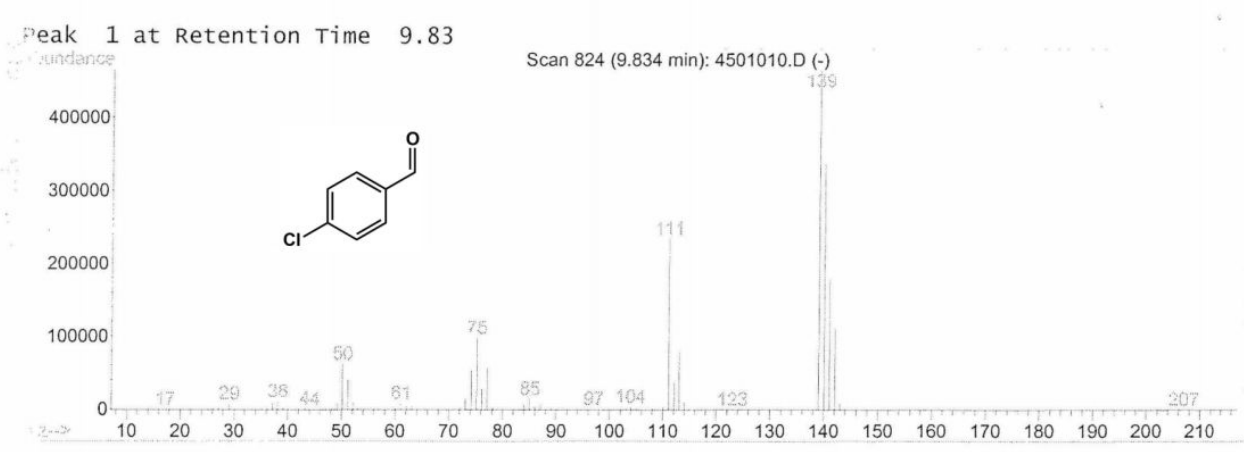

eak 2 at Retention Time 10.41

undance Scan 950 (10.406 min): 4501010.D (-)

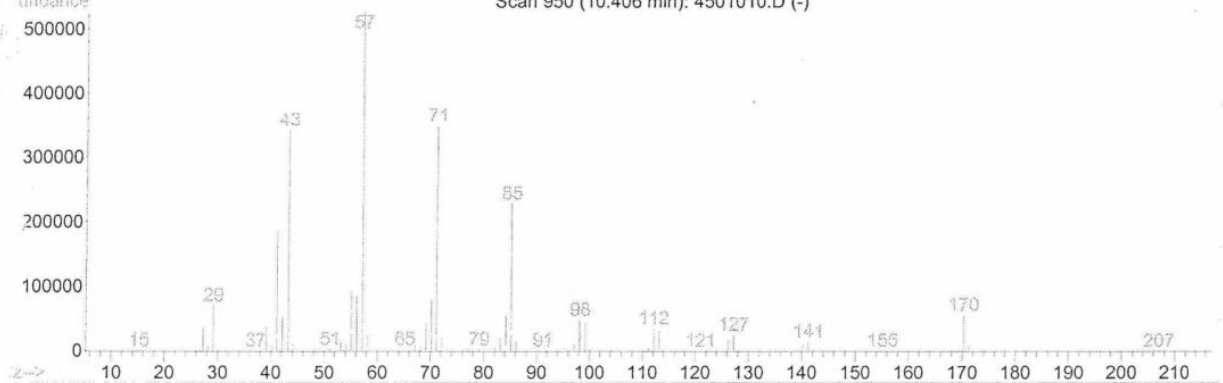

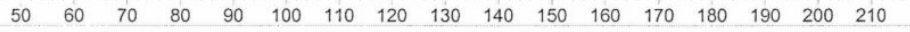

sak 3 at Retention Time 11.34
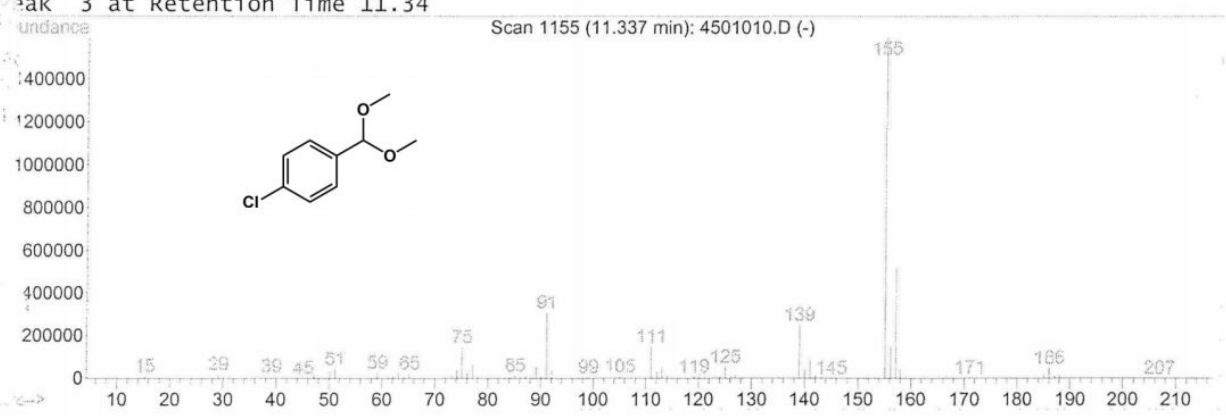

\DATA \19-09-11\4501010.D

Thu sep 12 16:19:49 2019

Page 2 of 2 


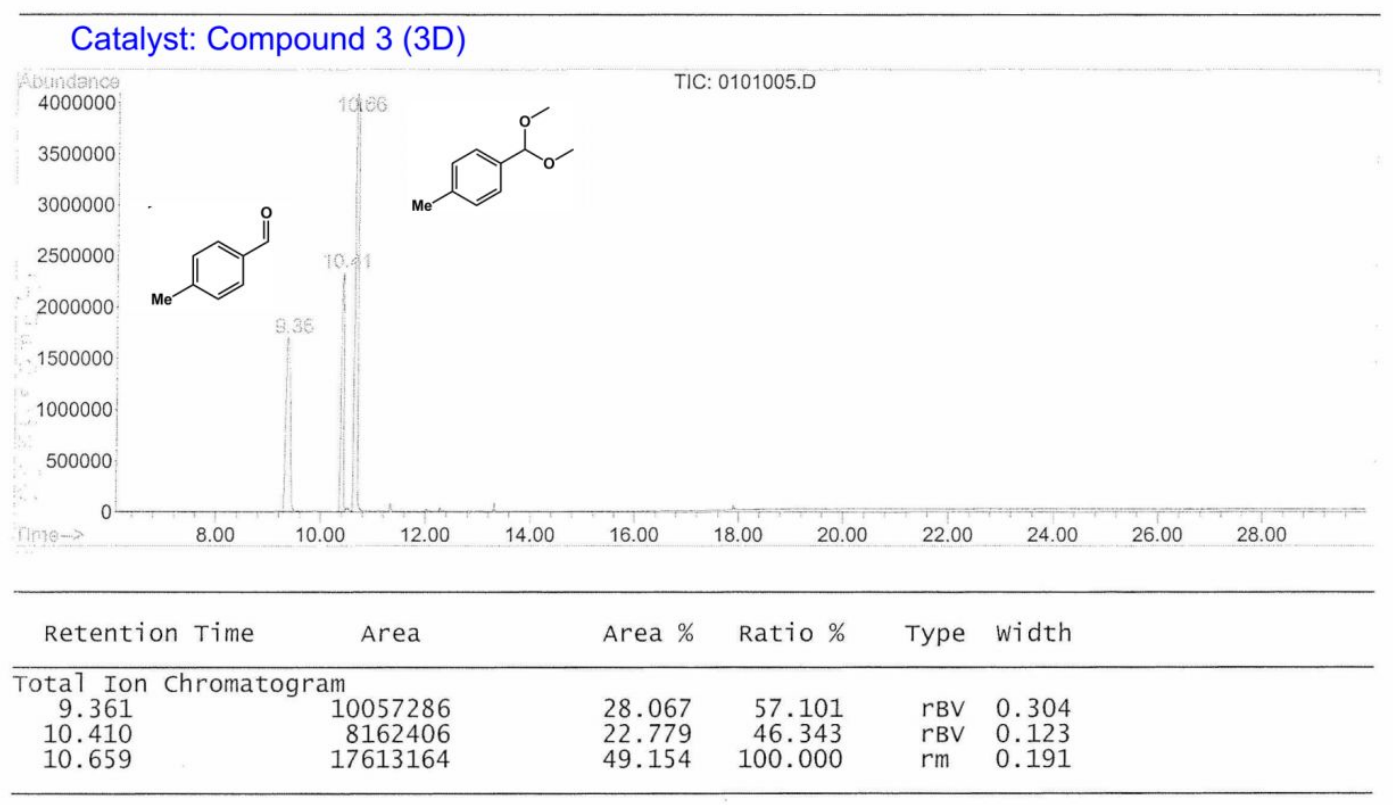




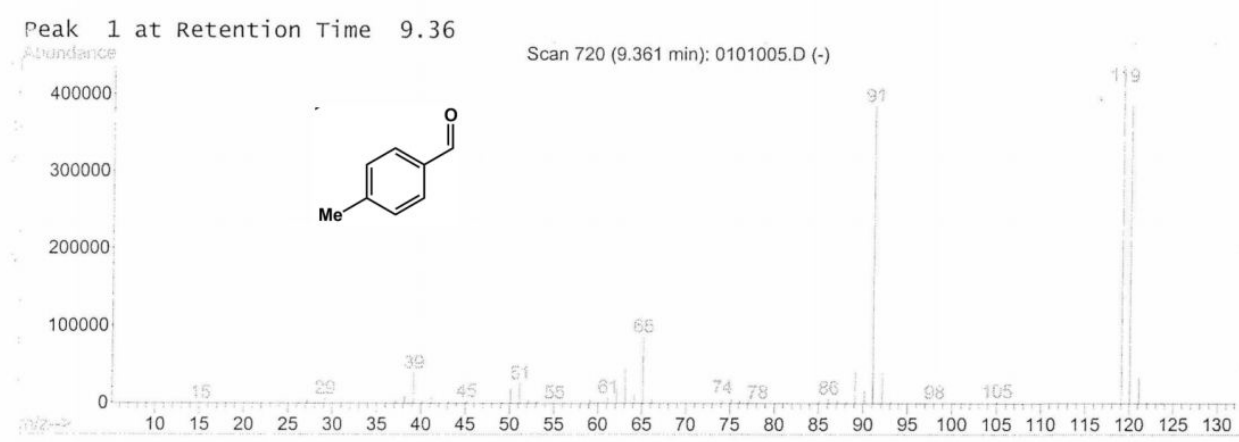

peak 2 at Retention Time 10.41

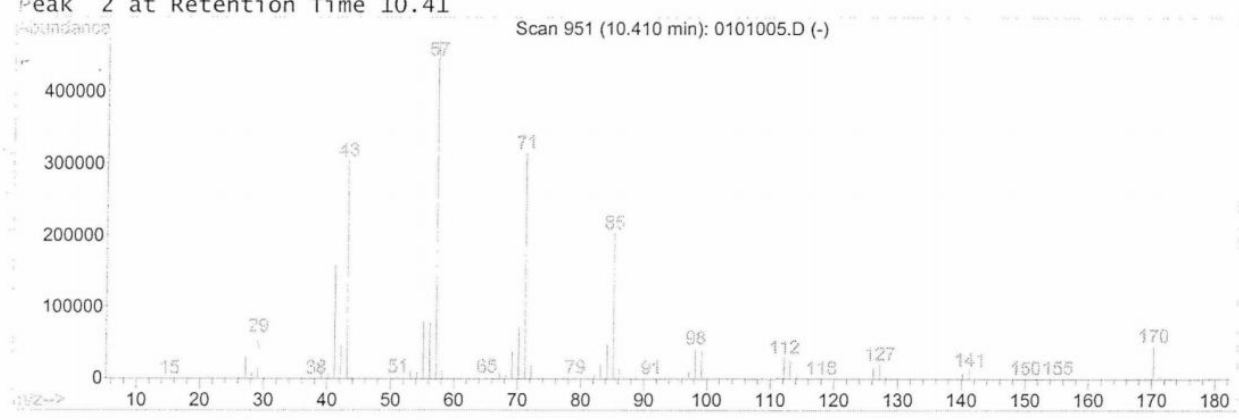

Peak 3 at Retention Time 10.66

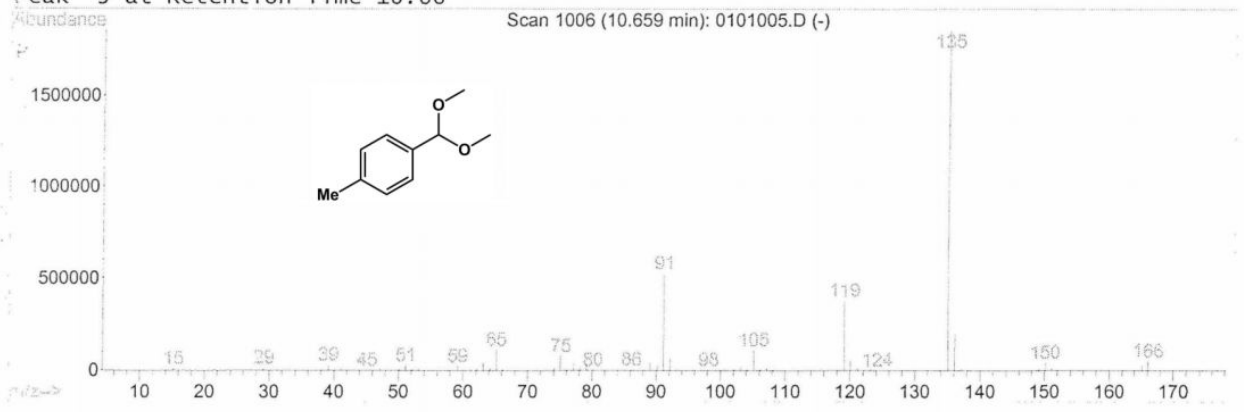




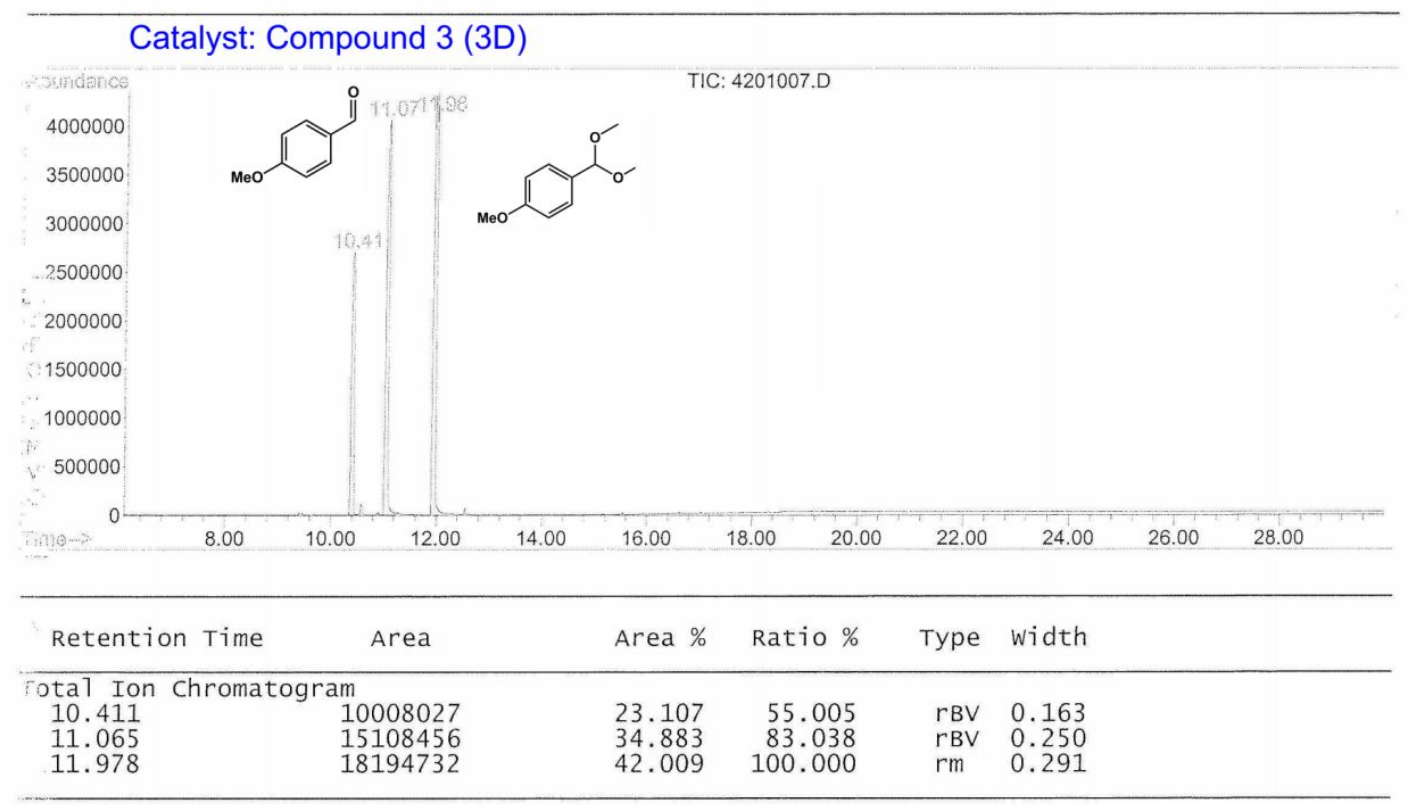

$\cdots$ 


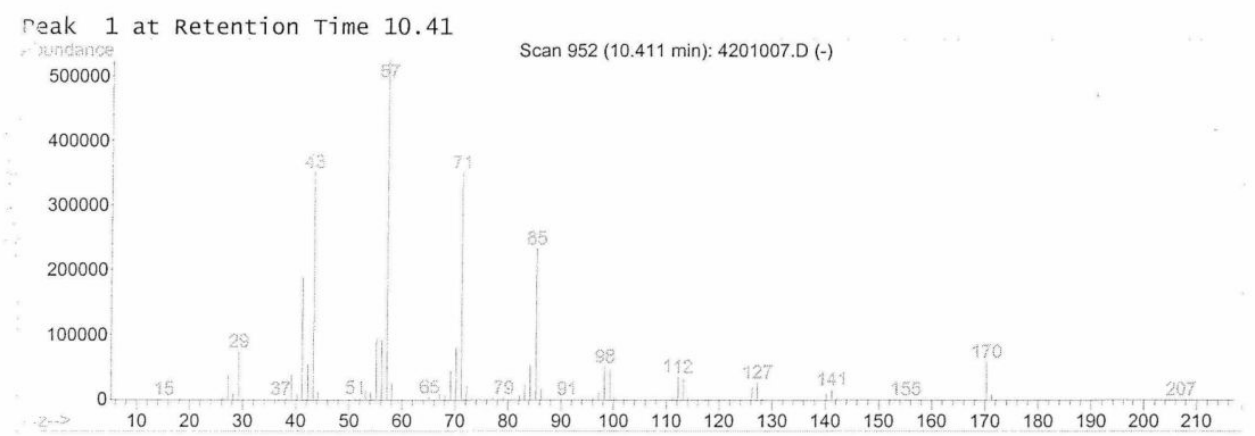

peak 2 at Retention Time 11.07

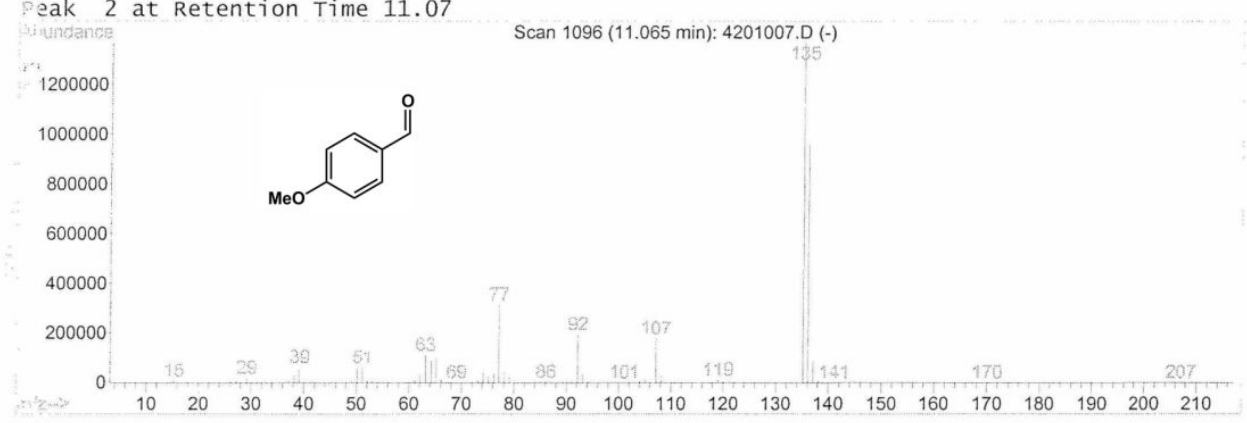

reak 3 at Retention Time 11.94

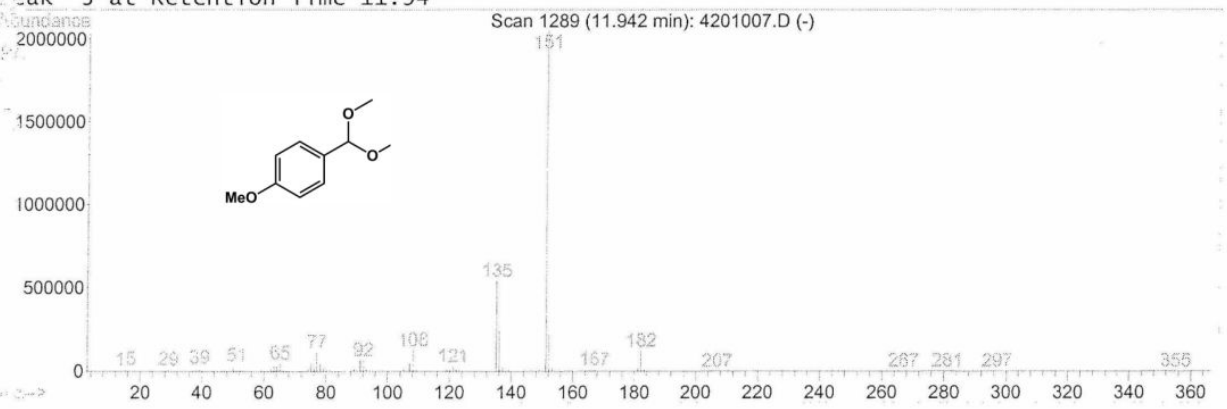

;is

$2^{2}$ 


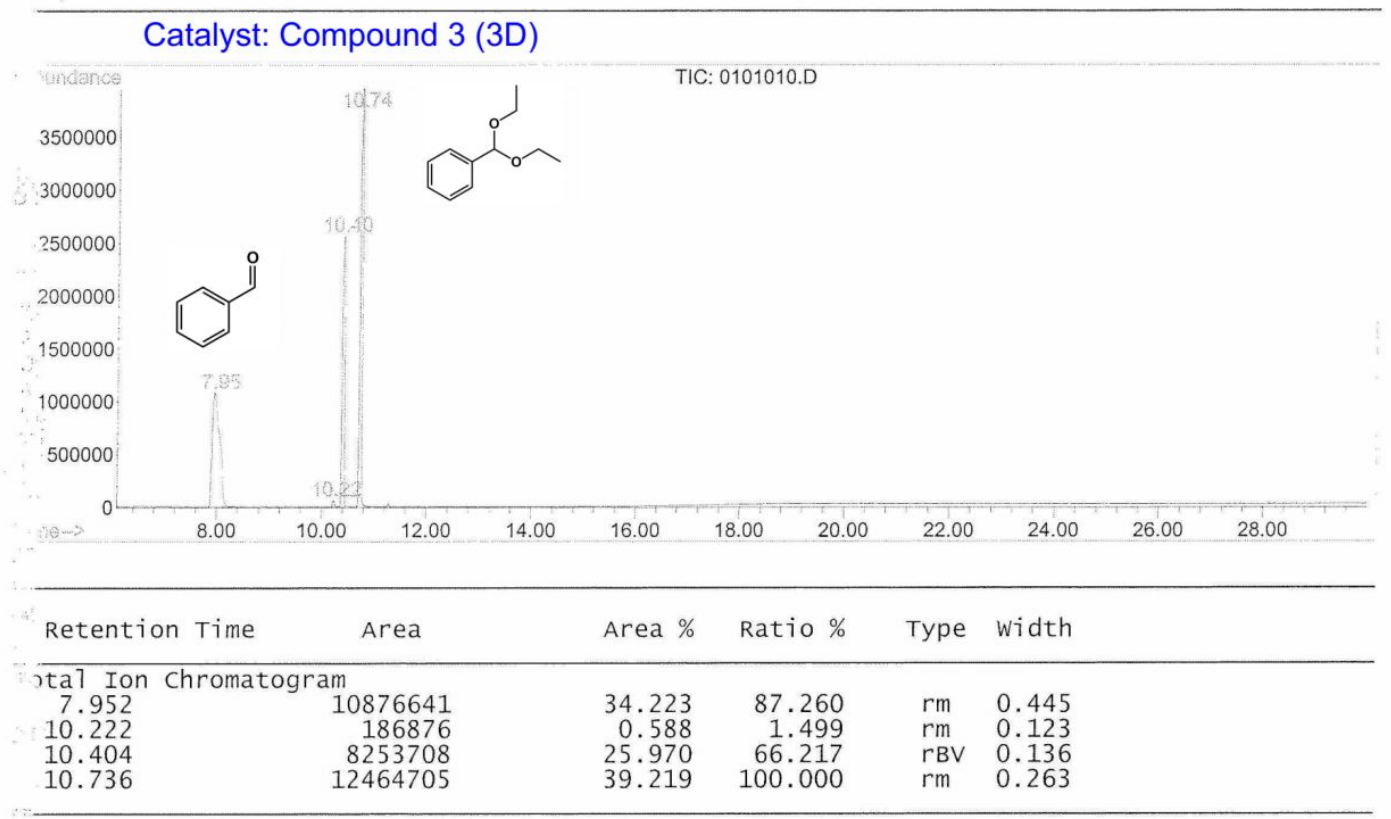




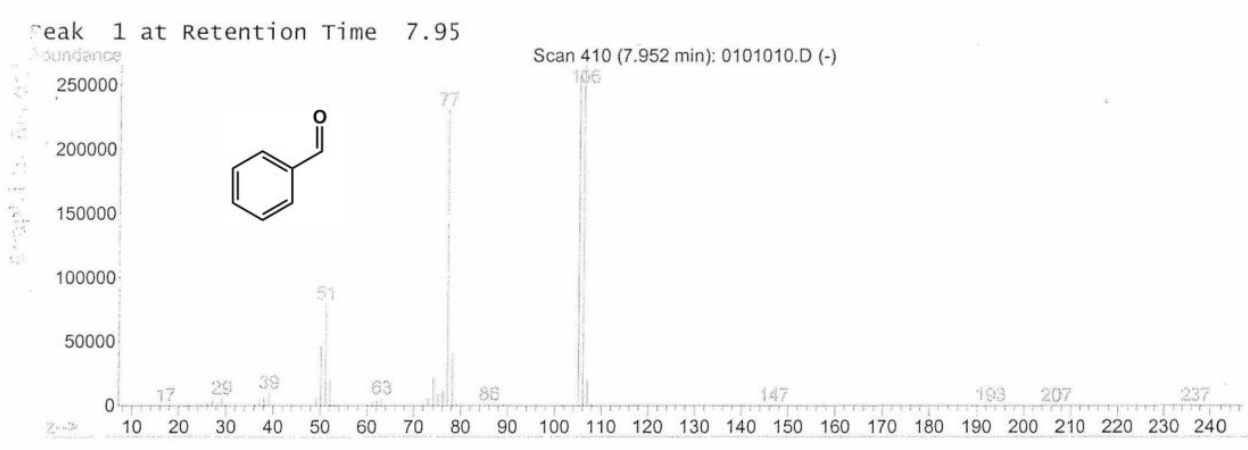

eak 2 at Retention Time 10.22

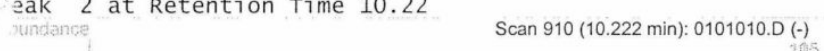

20000

15000

10000

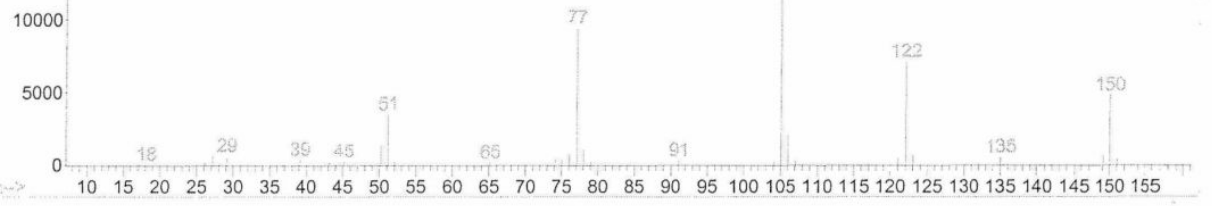

ak 3 at Retention Time 10.40

500000 Scan 950 (10.404 min): 0101010.D (-)

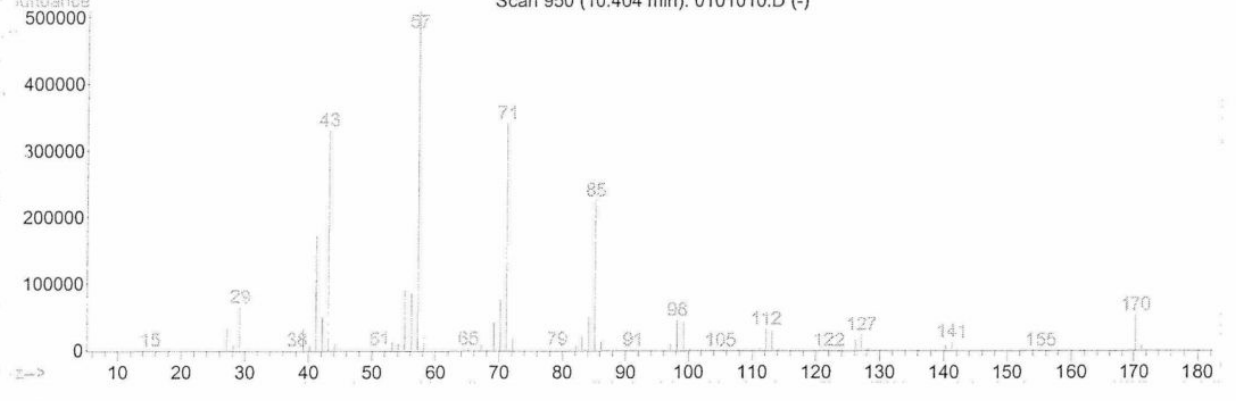

aak 4 at Retention Time 10.74

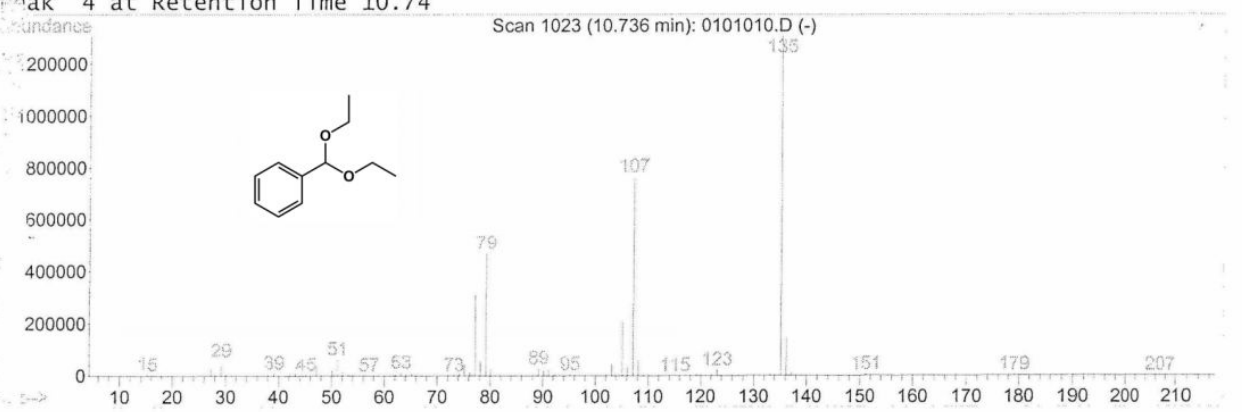

- \DATA \19-09-17\0101010.D

Thu Sep 19 10:15:42 2019

Page 2 of 2 


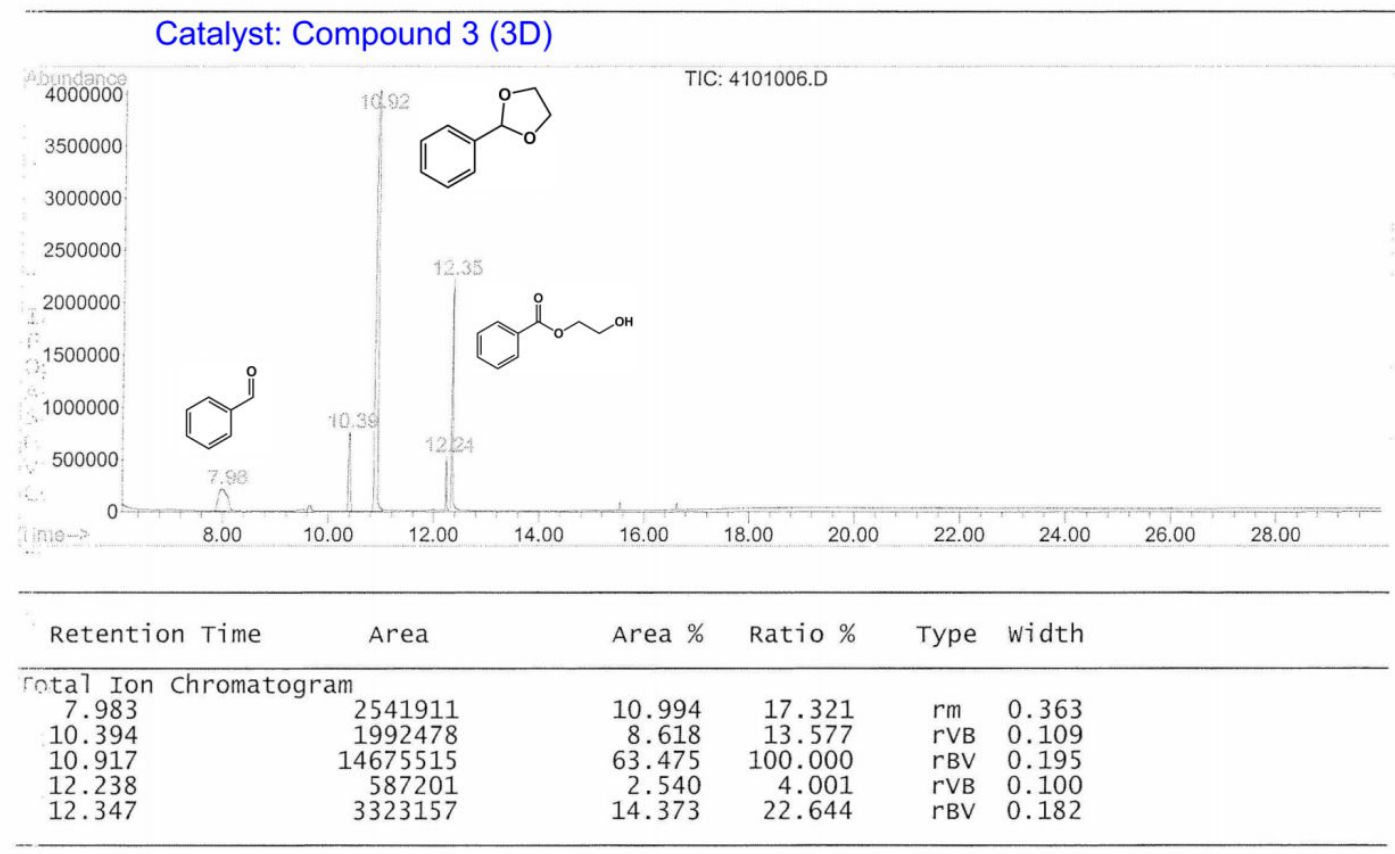

D: \DATA \19-09-11\4101006.D

Thu sep 12 16:17:35 2019

Page 1 


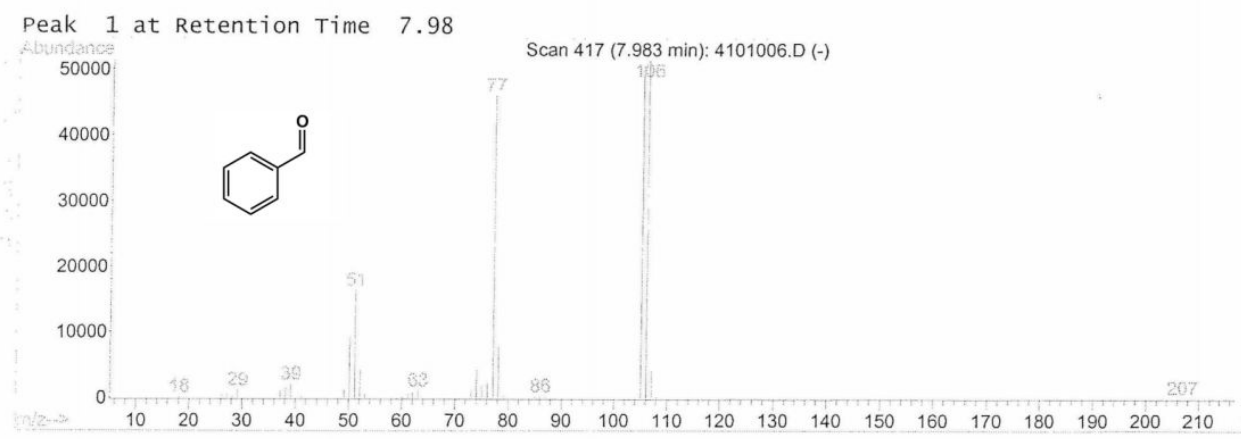

Peak 2 at Retention Time 10.39

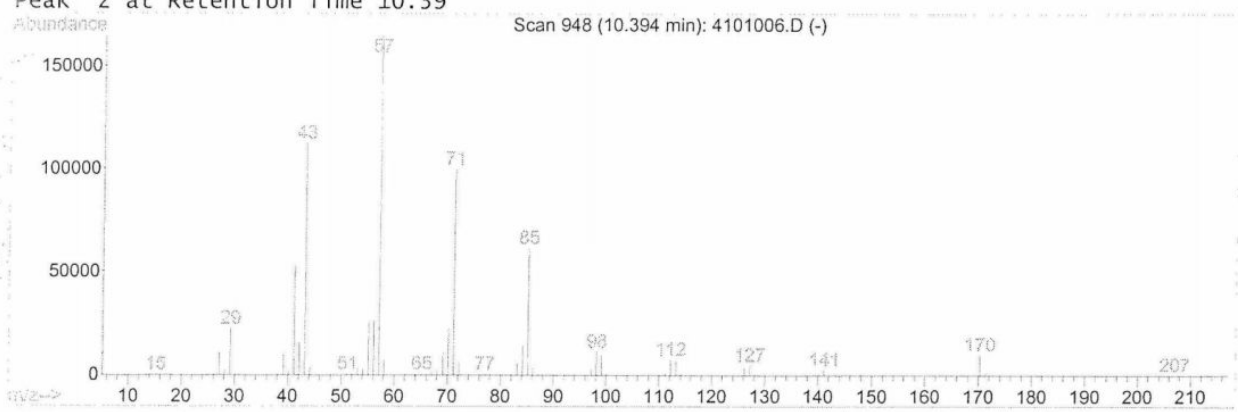

Peak 3 at Retention Time 10.92

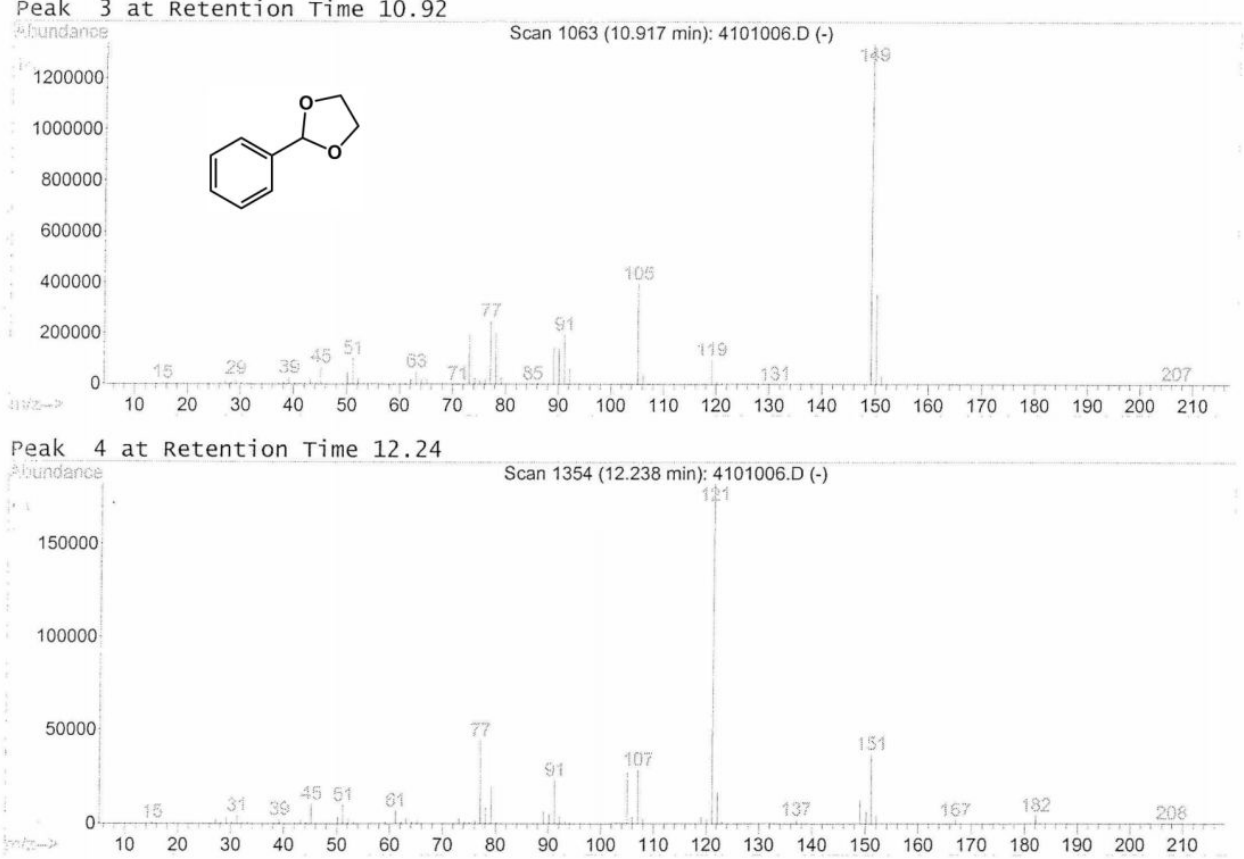

5: \DATA \19-09-11\4101006.D

Thu sep 12 16:17:25 2019

Page 2 of 3 


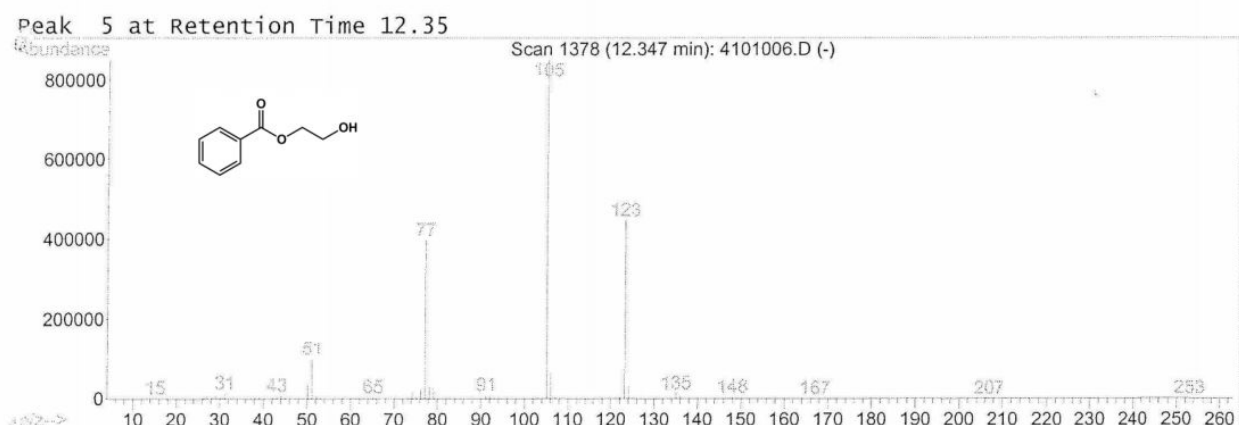




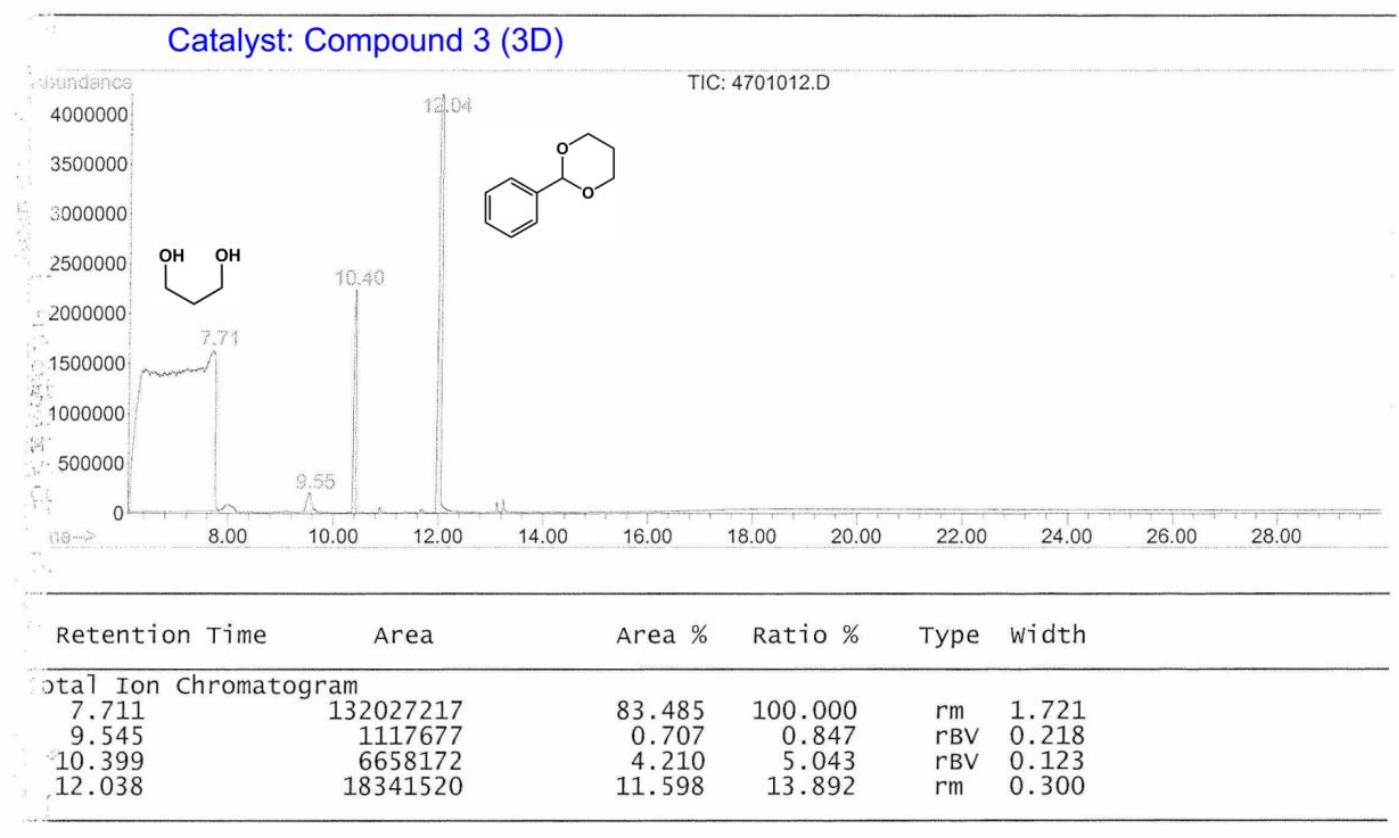

\DATA \19-09-11\4701012.D

Thu Sep 12 16:21:32 2019

Page 1 


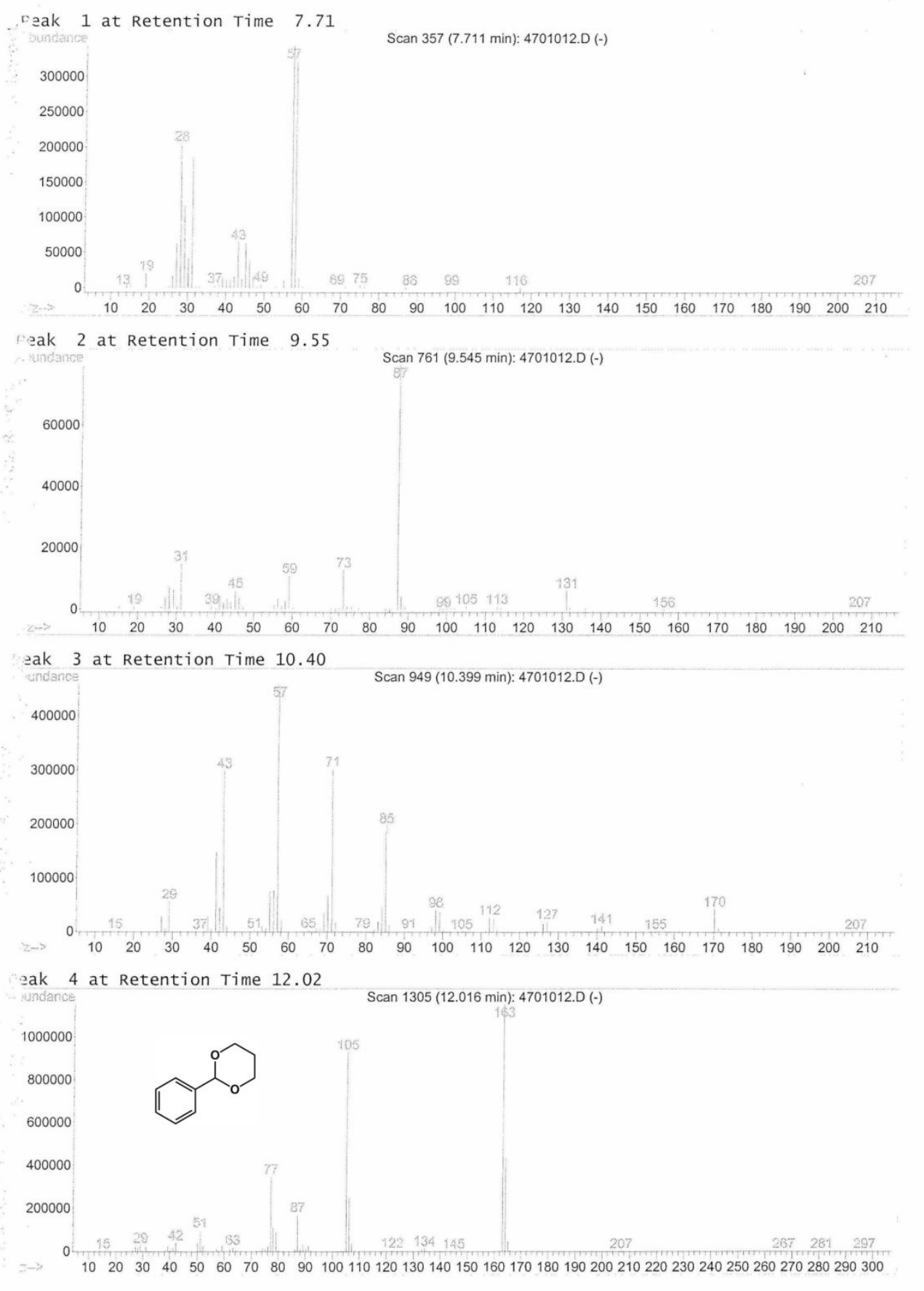

: \DATA \19-09-11\4701012.D

Thu sep 12 16:21:14 2019

Page 2 of 2 